Running Head: MOTIVATION VON LEHRKRÄFTEN

\title{
Motivation von Lehrkräften
}

\author{
Martin Daumiller \\ Universität Augsburg
}

Preprint-Version des Beitrags aus Enzyklopädie Erziehungswissenschaft Online (ISSN 2191-8325), Jahr 2018, Seite 1-31. doi:10.3262/EEO21180403

\begin{abstract}
Angaben zum Autor
Martin Daumiller, Lehrstuhl für Psychologie, Universität Augsburg, Universitätsstr. 10, 86135 Augsburg, Tel.: +49 821 598-5610, E-Mail: martin.daumiller@phil.uni-augsburg.de.
\end{abstract}




\begin{abstract}
Motivation von Lehrkräften gilt als wichtige Bedingung der Unterrichtsqualität, des Kompetenzzuwachses der Schüler(innen) sowie des Lernens und Wohlbefindens der Lehrkräfte. In den letzten Jahren ist dieses theoretische Konstrukt vermehrt in den Blick der empirischen Forschung gerückt und es wird vielfach mehr Forschung dazu gefordert. Es liegen verschiedene theoretische Konzepte und unterschiedliche Zugänge zur Motivation von Lehrkräften vor, zu denen der vorliegende Beitrag einen Überblick gibt und sie in ein übergeordnetes Modell einordnet. Durch diese Übersicht der Forschung zu Berufswahlgründen, Interesse, Selbstwirksamkeitserwartungen, der Zielorientierungstheorie sowie der Selbstbestimmungstheorie werden Bedingungsfaktoren, Korrelate und Folgen der Lehrkraftmotivationen expliziert, integrativ eingeordnet und kritisch diskutiert, was essenziell für deren empirische und praktische Nutzbarmachung ist.
\end{abstract}

Schlüsselbegriffe: Motivation, Lehrkräfte, Lehrer, Berufswahlgründe, Interesse, Selbstwirksamkeitserwartung, Zielorientierungstheorie, Zielstruktur, Selbstbestimmungstheorie 


\section{Inhaltsverzeichnis}

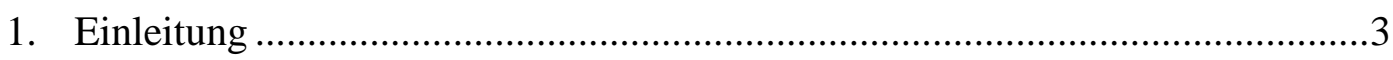

2. Definitionen, Konzeptualisierungen und Zugänge zum psychologischen Konstrukt der Motivation ............................................................................5

3. Rahmenmodell der Lern- und Leistungsmotivation von Lehrkräften...............6

4. Berufswahlgründe: internale und externale Gründe, FIT-Choice Theorie ........9

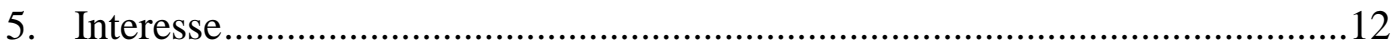

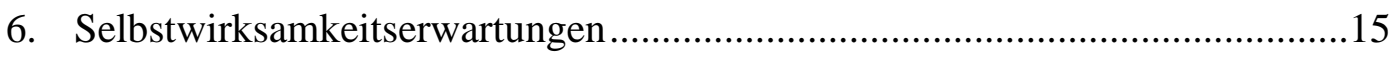

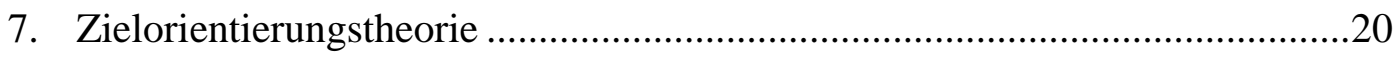

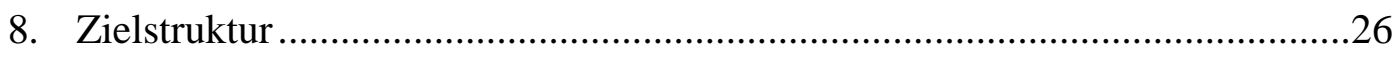

9. Intrinsische und extrinsische Motivation, Bedürfnisbefriedigung im Rahmen

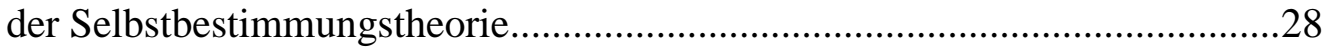

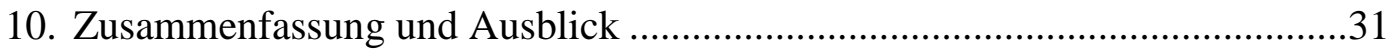

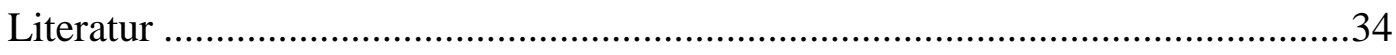




\section{Einleitung}

Die schul- und unterrichtsbezogene Bildungsforschung nahm in den letzten Jahrzehnten in Umfang und Qualität deutlich zu und wurde zunehmend auf innerschulische sowie unterrichtliche Bedingungsfaktoren und Prozesse fokussiert. Besonders im Zentrum stehen dabei Lehrkräfte (Terhart, Bennewitz \& Rothland, 2014), deren Kompetenzen, Kognitionen, Handeln und Affekt beschrieben, erklärt und unterstützt werden sollen.

Bereits in alltagspraktischer Sicht wird der Motivation von Lehrkräften eine Schlüsselrolle eingeräumt, was sich beispielsweise in Umfragen zu Merkmalen guter Lehrer(innen) oder Binsenweisheiten (z. B. ,motivierte Lehrkräfte machen guten Unterricht"“) widerspiegelt (Dresel \& Lämmle, 2017; Kunter, 2014). Auch in Modellen zum Lehrberuf nimmt Motivation einen prominenten Platz ein (z. B. Alexander, 2008; Baumert \& Kunter, 2011; Firestone \& Pennell, 1993; Shulman \& Shulman, 2004). Theoretisch wird angenommen, dass die Motivation von Lehrkräften mit der Qualität ihres Erlebens und Verhaltens im Beruf zusammenhängt und somit für den Lernzuwachs und die Motivation der Schüler(innen) bedeutsam ist (z. B. de Jesus \& Lens, 2005; Patrick et al. 2000; für einen Überblick siehe Richardson, Karabenick \& Watt, 2014). Darüber hinaus wird Motivation angesichts wandelnder Herausforderungen sowie im Rahmen lebenslangen Lernens als zentrale Bedingung erachtet (Spiel \& Schober, 2003). Aufgrund sich verändernder Schülerpopulationen oder curricularer Vorgaben ist es für Lehrkräfte auch nach Abschluss der formalen Ausbildung essenziell, ständig neues Wissen zu erwerben und vorhandenes anzupassen (Shulman \& Shulman, 2004) - wofür eine ausgeprägte Lernmotivation unabdingbar ist.

Ferner ist der Lehrberuf, und im Besonderen das Unterrichten selbst, auch für Lehrkräfte von vielfältigen Lern- und Leistungssituationen geprägt: In diesen gibt es facettenreiche Lern- und Leistungsanforderungen im täglichen und langfristigen Handeln, das in diverse soziale Leistungskontexte eingebettet ist (z. B. Lehrerkollegium, Elternschaft, Schülerschaft). Demnach wird Schule nicht nur für Schüler(innen), sondern auch für Lehrkräfte als ,,achievement arena“ (Butler, 2007, S. 242) erachtet. Nachdem der Lehrberuf darüber hinaus von relativ komplexen Tätigkeiten mit geringer externer Strukturierung und einem hohen Ausmaß erforderlicher Selbststeuerung geprägt ist (Lin, Schwartz \& Hatano, 2005; Sternberg \& Horvath, 1995), kann auch aus dieser Perspektive gefolgert werden, dass die Motivation der Lehrkräfte eine entscheidende Rolle für ihr berufliches Erleben und Verhalten einnimmt.

Tatsächlich ist die Motivation von Lehrkräften in jüngster Zeit in den Blick der empirischen Forschung gerückt (,Zeitgeist of interest“", Watt \& Richardson, 2008). 
Theorien der Lern- und Leistungsmotivation, die zuvor primär an Schüler(inne)n und Studierenden angewendet wurden, fanden hier fruchtbaren Boden (Richardson et al., 2014). Ausgehend von primär eindimensionalen Fragen danach, wie sehr Lehrkräfte motiviert sind und aus welchen Gründen sie sich für die Lehrprofession entscheiden, hat sich die Lehrkraftmotivationsforschung in letzter Zeit verstärkt der Frage zugewendet, welche motivationalen Merkmale den Umfang und die Qualität des Erlebens und Verhaltens von Lehrkräften bestimmen (vgl. Kunter, 2014).

So wie es auch in der übergreifenden Motivationsforschung unterschiedliche theoretische Zugänge und nicht immer klar voneinander abgegrenzte Konzepte gibt (,fuzzy but powerful constructs“; Pintrich, 1994, S. 139), liegen auch verschiedene theoretische Konzepte und unterschiedliche Zugänge zur Motivation von Lehrkräften vor, was wiederum das Verständnis und die Nutzbarmachung dieses wichtigen Forschungsbereichs einschränkt. Die klare Artikulation der Natur eines Motivationskonstrukts und seine Abgrenzung und Verortung gegenüber anderen ist daher essenziell (vgl. Murphy \& Alexander, 2000; Wigfield \& Cambria, 2010). Es gibt jedoch bislang kein übergreifendes Framework oder Modell dazu: In den wenigen existierenden theoretischen Modellen zur Motivation von Lehrkräften wird diese meist primär leistungsthematisch interpretiert und auf das instruktionale Handeln im Unterricht fokussiert (z. B. de Jesus \& Lens, 2005).

Daran setzt vorliegender Beitrag an. Er hat zum Ziel, einen Überblick über die Forschungsarbeiten zum theoretischen Konzept der Motivation von Lehrkräften zu geben, indem die verschiedenen Zugänge theoretisch erläutert und unterschieden sowie in einem übergreifenden Modell zur Lehrkraftmotivation verortet werden. Durch eine Übersicht der bisherigen empirischen Forschungsbefunde zu den unterschiedlichen theoretischen Zugängen werden Bedingungsfaktoren, Korrelate und Folgen der Lehrkraftmotivation expliziert, was essenziell für die empirische und praktische Nutzbarmachung dieses Konstrukts ist.

Dazu wird zunächst ein Überblick über das psychologische Konstrukt der Motivation gegeben (Abschnitt 2) und darauf aufbauend ein Rahmenmodell zur Motivation von Lehrkräften vorgestellt (Abschnitt 3). Dieses verortet etablierte Konstrukte, die in der Forschung zur Lehrkraftmotivation Anwendung finden und spezifiziert deren theoretische Zusammenhänge mit Bedingungsfaktoren und Auswirkungen der Lehrkraftmotivation. Anschließend erfolgt eine Übersicht über die verschiedenen theoretischen Konstrukte und deren dazugehörigen empirischen Forschungsbefunde (Abschnitte 4-9), die schließlich resümiert und in Hinblick auf zukünftige Forschungsrichtungen in diesem verhältnismäßig neuen Forschungsfeld diskutiert werden (Abschnitt 10). 


\section{Definitionen, Konzeptualisierungen und Zugänge zum psychologischen Konstrukt der Motivation}

„Motivation“" (lat. movēre, jmd. oder etw. bewegen) charakterisiert Grund, Absicht und Richtung menschlichen Handelns. Dieses hypothetische (d. h. nicht direkt beobachtbare oder messbare) Konstrukt beschrieb man bereits in frühen psychologischen Theorien menschlichen Erlebens und Verhaltens Ende des 19. Jahrhunderts (vgl. Eccles \& Wigfield, 2002). Seitdem veränderten sich die Definitions- und Erklärungsansätze je nach theoretischen Strömungen und zeitgenössischen Überzeugungen.

Zunächst lag ein eindimensionales Motivationskonzept vor, in dem primär die Handlungsinitiierung fokussiert wurde. Dies deckt sich mit typischen

Alltagsverständnissen und auch den ersten Forschungsarbeiten zur Motivation von Lehrkräften. Motivation wurde darin als eine oft nicht näher ausdifferenzierte Art innerer Energie aufgefasst. Von dieser wurde angenommen, dass sie in ihrer Stärke variiert und somit beispielsweise bestimmt, wie engagiert Lehrkräfte ihren Beruf ausüben; während sie von außen durch Anreizstrukturen des Lehrberufs beeinflusst werden kann (Firestone \& Pennell, 1993; Lortie, 1975; Shulman \& Shulman, 2004; zsf. Kunter, 2014).

Seit den 1980er Jahren ist in der psychologischen Motivationsforschung dieses eindimensionale Verständnis weiter ausdifferenziert worden (Mayer, Faber \& Xu, 2007; Pintrich, 2003; Schunk, Meece \& Pintrich, 2013). Im Rahmen eines neueren und breiteren Verständnisses wird angenommen, dass Motivation den gesamten Handlungsprozess umfasst, also bei der Planung, Ausführung und Evaluation von Handlungen zum Tragen kommt (Heckhausen, 1987; Heckhausen \& Gollwitzer, 1987), und sowohl von Merkmalen der Person als auch von Merkmalen der spezifischen Situation bzw. des Kontextes abhängt (vgl. Mitchell, 1997; Rheinberg, 2008). Obwohl sich Individuen untereinander also hinsichtlich einer Reihe stabiler motivationaler Tendenzen und Überzeugungen unterscheiden, müssen einzelne Personen keineswegs in jeder Situation ähnlich motiviert sein. Folglich ist es wichtig, die aktuelle Motivation in einer spezifischen Lern- und Leistungssituation getrennt von motivationalen Tendenzen sowie personellen und kontextuellen Bedingungsfaktoren zu betrachten.

Im Rahmen dieses aktuellen Verständnisses von Motivation steht nicht mehr die Frage im Vordergrund, wie sehr eine Person motiviert ist, sondern auf welche Art und Weise sie motiviert ist. Diese Frage nach der Qualität von Motivation ist schließlich insbesondere in lern- und leistungsthematischen Settings wie dem Unterrichten maßgeblich, um Erleben und Verhalten zu beschreiben (Pintrich, 2000a, 2000b). Es kann angenommen werden, dass Lehrkräfte je nach Qualität 
ihrer aktuellen Motivation beispielsweise mehr oder weniger Energie in die Unterrichtsvorbereitung investieren und gegebenenfalls zusätzliche Lernangebote aufsuchen (behaviorale Unterschiede), Hilfe unterschiedlich einschätzen (kognitive Unterschiede) sowie Belastungsstressoren anders erleben (affektive Unterschiede). Dieses ausdifferenzierte Verständnis von Motivation ist in der Forschung zu Lehrkräften erst seit den 2000er Jahren zunehmend zu finden.

Eine Betrachtung der Motivation ist entsprechend auf verschiedenen Hierarchieebenen möglich, je nachdem, ob überdauernde Personenmerkmale oder spezifische Unterschiede in einer aktuellen Situation im Vordergrund stehen. Nachdem es sich um ein hypothetisches Konstrukt handelt, gibt es darüber hinaus unterschiedliche theoretisch-konzeptuelle Zugänge, um die Intensität und Qualität von Motivation zu beschreiben und zu verstehen. Vor allem seit der kognitiven Wende der Psychologie in den 1960er Jahren entwickelten sich mehrere sozialkognitive Motivationstheorien (vgl. Dember, 1974; Pervin, 1992), die in der jüngsten Zeit auch auf Lehrkräfte angewendet wurden und aktuell zu den in diesem Forschungsbereich etablierten Zugängen zählen. Dazu gehören das Konzept der Selbstwirksamkeitserwartung (Bandura, 1977, 1997), die ErwartungsWert-Theorie (Eccles, 1983), die Attributionstheorie (Weiner, 1985), die Selbstbestimmungstheorie (Deci \& Ryan, 1985, 1993) und die Zielorientierungstheorie (Dweck, 1986; Nicholls, 1984). Diese verschiedenen Zugänge sind nicht immer klar voneinander abgegrenzt und überlappen sich (Pintrich, 1994). Zu einem adäquaten Verständnis von Motivation müssen daher die verschiedenen Zugänge integrativ berücksichtigt werden. Dazu wird im folgenden Abschnitt ein Rahmenmodell zur Lehrkraftmotivation vorgestellt, in dem diese unterschiedlichen Zugänge verortet werden können.

\section{Rahmenmodell der Lern- und Leistungsmotivation von Lehrkräften}

Abbildung 1 zeigt ein Rahmenmodell zur integrierten Darstellung von Komponenten, Bedingungen und Konsequenzen der Lern- und Leistungsmotivation von Lehrkräften. 


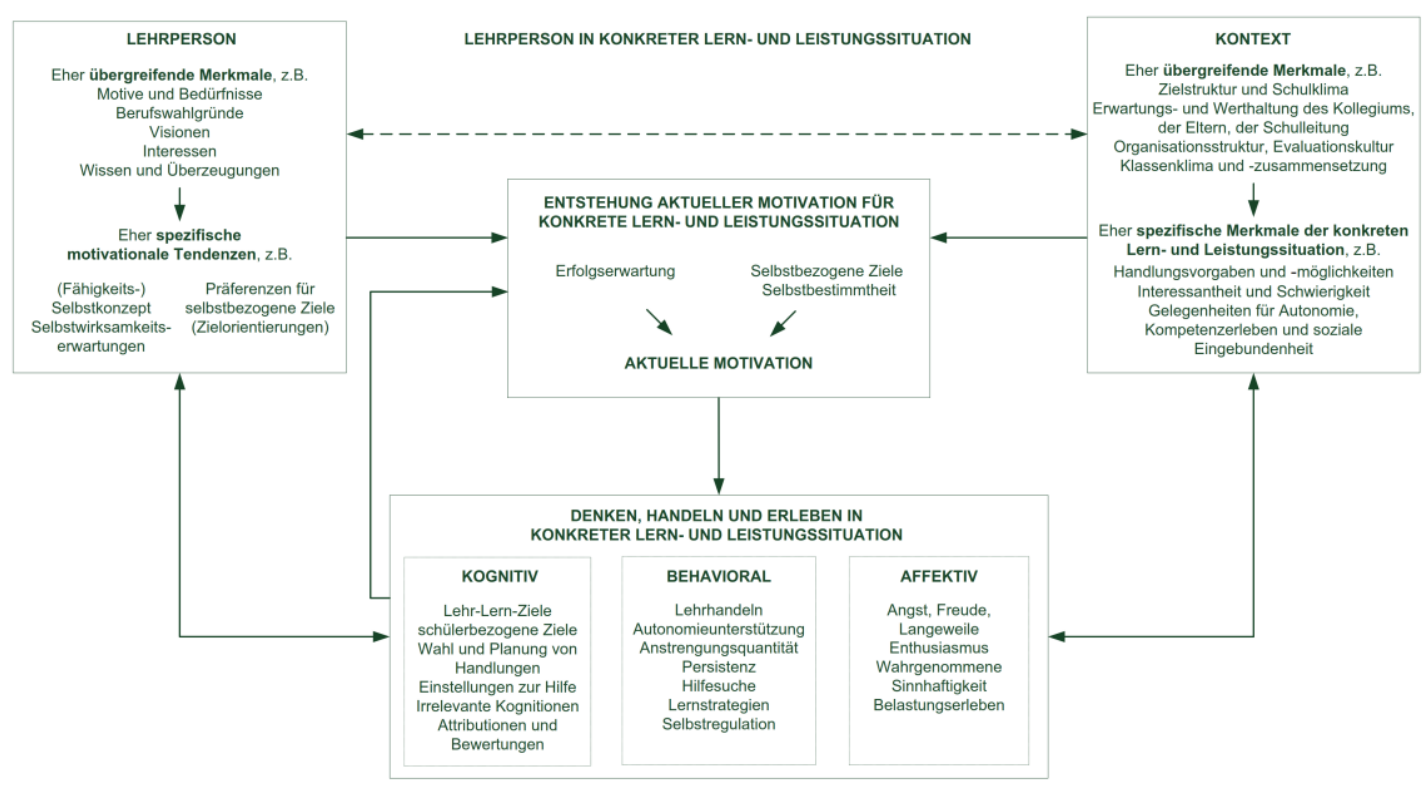

Abbildung 1. Rahmenmodell der Lern und Leistungsmotivation von Lehrkräften (modifiziert nach Dresel \& Lämmle, 2017)

Im Kern dieses Modells steht die aktuelle Motivation in einer spezifischen Lernund Leistungssituation. Diese besteht aus Erwartungen hinsichtlich der Realisierbarkeit (Erwartungskomponente) und der Wünschbarkeit (Wertkomponente) von Handlungsoptionen. Diese wiederum sind von eher erwartungsbezogenen (z. B. Selbstwirksamkeitserwartungen, Selbstkonzept; vgl. Abschnitt 3) und eher wertbezogenen (z. B. Präferenzen für selbstbezogene Ziele; vgl. Abschnitt 4) motivationalen Tendenzen abhängig, die - ebenso wie die aktuelle Motivation - von Merkmalen der Person sowie von Merkmalen des Kontexts abhängig sind. Das zentrale Zusammenspiel von Erwartung und Wert deckt sich mit vielen Erwartungs-Wert-Modellen der Motivation (z. B. Eccles, 1983, 2005, 2009). Diese gehen davon aus, dass die resultierende Motivation mit Verbesserungen bei jeder der beiden Komponenten ansteigt (jedoch keine davon hinreichend ist; d. h. bei einer Erfolgserwartung von Null hat auch ein hoher wahrgenommener Wert keine aktivierende Funktion) und diese von Bewertungen früherer Lern- und Leistungserfahrungen, der sozialen Umwelt sowie motivationalen Tendenzen abhängen (vgl. Dresel \& Lämmle, 2017). Hinsichtlich letzterer stellen Selbstwirksamkeitserwartungen und Präferenzen für selbstbezogene Ziele in der Forschung zu motivationalen Tendenzen bei Lehrkräften besonders häufig verwendete theoretische Zugänge dar, weswegen in den Abschnitten 3 und 4 ausführlich auf diese eingegangen wird. 
$\mathrm{Zu}$ weiteren, übergreifenden Personenmerkmalen zählen vergleichsweise globale und überdauernde Aspekte, wie Motive und Bedürfnisse, Berufswahlgründe (vgl. Abschnitt 4), Visionen und Leidenschaft sowie Wissen und Überzeugungen. Andererseits gehören dazu auch Aspekte, die oft erst in einem bestimmten Kontext aktiviert werden, wie etwa das Interesse (vgl. Abschnitt 5). Dies illustriert auch den im Rahmenmodell durch den Doppelpfeil dargestellten Zusammenhang zwischen Personen- und Kontextmerkmalen: So ist die Entwicklung von Interessen beispielsweise unter anderem von der Anreizstruktur des Kontexts abhängig, allerdings auch dafür verantwortlich, wie der Lehrberuf innerhalb der Lehrerschaft wahrgenommen und bewertet wird.

$\mathrm{Zu}$ den Kontextmerkmalen zählen einerseits recht globale und überdauernde Merkmale wie die Zielstruktur (vgl. Abschnitt 8) und das Klima in der Schule und in einzelnen Klassen, die Erwartungs- und Werthaltungen des Kollegiums, der Eltern und der Schulleitung, die Unterstützung durch Kolleg(inn)en, die Organisationsstruktur sowie die Evaluationskultur der Einrichtung. Andererseits sind die (davon abhängigen) Merkmale der spezifischen Lern- und Leistungssituation zu nennen. Dazu zählen etwa die konkreten Handlungsvorgaben und -möglichkeiten, die Interessantheit und Schwierigkeit eines Lehrinhalts oder -kontexts und das Ausmaß an Autonomie, Kompetenzerleben und sozialer Eingebundenheit - die zu einer entsprechenden Befriedigung psychologischer Grundbedürfnisse führen und somit einen bedeutsamen Einfluss auf das Ausmaß an Selbstbestimmung der aktuellen Motivation nehmen können (vgl. Abschnitt 9).

Das Modell beinhaltet darüber hinaus das Erleben und Verhalten der Person in der konkreten Lern- und Leistungssituation. Hier werden der aktuellen Motivation unterschiedliche kognitive, behaviorale und affektive Konsequenzen zugeschrieben. Je nach Intensität und Qualität der aktuellen Motivation ist anzunehmen, dass sich die Lehrkräfte divergierende (didaktische) Lehr-Lern-Ziele oder unterschiedliche schülerbezogene Ziele setzen. Außerdem sind Unterschiede in der Handlungsauswahl und -planung sowie deren Bewertung denkbar (etwa hinsichtlich der Nützlichkeit von Hilfesuche) und ein unterschiedliches Ausmaß handlungsirrelevanter Kognitionen plausibel. Ferner wird die aktuelle Motivation für unterschiedliches Verhalten verantwortlich gemacht - etwa in Form des konkreten unterrichtlichen Handelns (bspw. in Form spezifischer Lehrstrategien, -schwerpunkte und -inhalte), aber auch für die Intensität und die Persistenz, mit der geplante Handlungen ausgeführt und auf welche Art und Weise diese Handlungen bei Schwierigkeiten angepasst und modifiziert werden. Darüber hinaus wird die aktuelle Motivation mit unterschiedlichem affektiven Erleben und Bewerten der spezifischen Lern- und Leistungssituation in Verbindung gebracht. Hierzu sind beispielsweise das Erleben von Lern- und Leistungsemotionen, 
Enthusiasmus sowie das Belastungserleben anzuführen. Über diese drei Bereiche vermittelt, sind schließlich auch Effekte auf die Ergebnisse des pädagogischen Handelns, die Schüler(innen) und deren Motivation sowie Kompetenzzuwachs anzunehmen. Daran schließt sich die Bewertung der Lern- und Leistungshandlungen an, bei der die Ergebnisse als erfolgreich oder misslungen bewertet und auf unterschiedliche Ursachen zurückgeführt werden (Attributionsprozesse). An dieser Stelle ist es wichtig festzuhalten, dass auch die übergeordneten Personen- und Kontextmerkmale Einfluss auf die eben dargestellten Prozesse nehmen (z. B. beeinflussen Umweltmerkmale wie die vorherrschenden Evaluationsmaßstäbe die Bewertung von Handlungsergebnissen; und Wissen und Überzeugungen sind mitverantwortlich für das konkrete instruktionale Handeln). Außerdem wirken die Bewertung und das Erleben von Handlungsergebnissen zurück auf die aktuelle Motivation, die motivationalen Tendenzen und die Personen- und Kontextmerkmale (s. a. Schunk et al., 2013).

Die Forschungsliteratur zur Motivation von Lehrkräften verwendet häufig Berufswahlgründe, Interessenstheorien, Selbstwirksamkeitserwartungen, die Zielorientierungstheorie und die Selbstbestimmungstheorie. Die folgenden Abschnitte stellen diese daher theoretisch dar und beschreiben zugehörige empirische Forschungsergebnisse, die in Zusammenschau die zuvor theoretisch abgeleiteten Auswirkungen auf Kognition, Handeln und Affekt im Beruf illustrieren.

\section{Berufswahlgründe: internale und externale Gründe, FIT-Choice Theorie}

Ausgangspunkt vieler Arbeiten im Bereich der Lehrkraftmotivation ist die Annahme, dass Lehrkräfte aufgrund der Besonderheiten ihres Berufs bereits eine besonders günstige Motivation haben sollten. Besonders in frühen Arbeiten zu diesem Themenkomplex wurden dazu die Berufsanreize analysiert (zsf. Kunter, 2014). Entsprechende Forschungsarbeiten berichteten geringe extrinsische Anreize (z. B. relativ flacher Karriereverlauf, moderat hohes Einstiegsgehalt aber nur wenig Aufstiegsmöglichkeiten, Gehaltsverbesserungen oft nicht unmittelbar mit höheren Anstrengungen verbunden; s. a. Terhart, 2000). Gleichzeitig wurden jedoch auch nur eingeschränkte intrinsische Anreize berichtet (z. B. Erfolge oft nur zeitversetzt sichtbar und Sorge um geringe Anerkennung von der Öffentlichkeit, s. a. Osterwalder, 2003). Aktuellere Studien, in denen unter anderem die Anreizstruktur des Lehrberufs mit ähnlichen Professionen verglichen wurde, ziehen demgegenüber ein differenzierteres Bild und verweisen auf deutliche extrinsische (bspw. langfristige finanzielle Erträge hinsichtlich der Altersvorsorge, 
der Sicherheit des Arbeitsplatzes sowie der Familienfreundlichkeit) sowie intrinsische Anreize (bspw. die steigende Wertschätzung des Lehrberufs in Deutschland). Dies verweist darauf, dass der Lehrberuf nicht per se weniger befriedigend ist als andere Berufe und nicht davon ausgegangen werden sollte, dass Lehrkräfte „mehr“ oder „,bessere“ Motivation als andere Berufstätige brauchen, um im Beruf zu verbleiben und darin erfolgreich zu agieren (Kunter, 2014). Gleichwohl gibt es, auch über verschiedene Schulformen hinweg, beachtliche interindividuelle Unterschiede seitens der Lehrkräfte in der Wahrnehmung und Bewertung des Lehrberufes sowie den Gründen, einen entsprechenden Werdegang einzuschlagen.

Diese Unterschiede wurden explizit in Studien zu Berufswahlgründen von Lehrkräften und Lehramtsstudierenden aufgegriffen. Darin wird angenommen, dass Personen bevorzugt Kontexte aufsuchen und - vermittelt über die Bewertung der Wünschbarkeit und Erreichbarkeit der verschiedenen Handlungsoptionen Verhaltensweisen zeigen, die zur Befriedigung ihrer Berufswahlgründe beitragen. Zu solchen Verhaltensweisen zählt die Berufswahl, aber auch spezifisches späteres Handeln im Beruf.

Ausgehend von früheren Arbeiten, die häufig keine einheitliche theoretische Basis hatten, bewährte sich in dieser Forschungsrichtung ein Zugang basierend auf Erwartungs-Wert-Modellen der Motivation, um einen systematischen und einheitlichen theoretisch fundierten Zugang zu den Berufswahlgründen von Lehrkräften zu ermöglichen (z. B. Pohlmann \& Möller, 2010; Richardson \& Watt, 2006; Watt \& Richardson, 2007). Von Watt und Richardson wurde beispielsweise ein integratives und erweitertes Modell basierend auf der Erwartungs-WertTheorie von Eccles $(2005,2009)$ vorgeschlagen (Factors Influencing Teaching Choice, FIT-Choice; zsf. Watt \& Richardson, 2008). Dieses Modell inkludiert die zuvor bereits aufgeführten Gründe für die Berufswahl, jedoch auch fähigkeitsbezogene Überzeugungen, die in vorherigen Arbeiten zur Berufswahlmotivation von Lehrkräften oft vernachlässigt wurden. Die Autor(inn)en gehen davon aus, dass die Wahl für den Lehrberuf von vorangeschalteten sozialen Einflüssen (z. B. Zu- oder Abraten von anderen Personen) sowie Lehr- und Lernerfahrungen abhängt, gefolgt von den proximaleren Einflüssen der eingeschätzten (a) Anforderungen und Vorteilen des Lehrberufs, (b) der eigenen Kompetenz als Lehrkraft, (c) dem Wert des Lehrberufs sowie (d) der Berufsalternativen (vgl. Abbildung 2). 


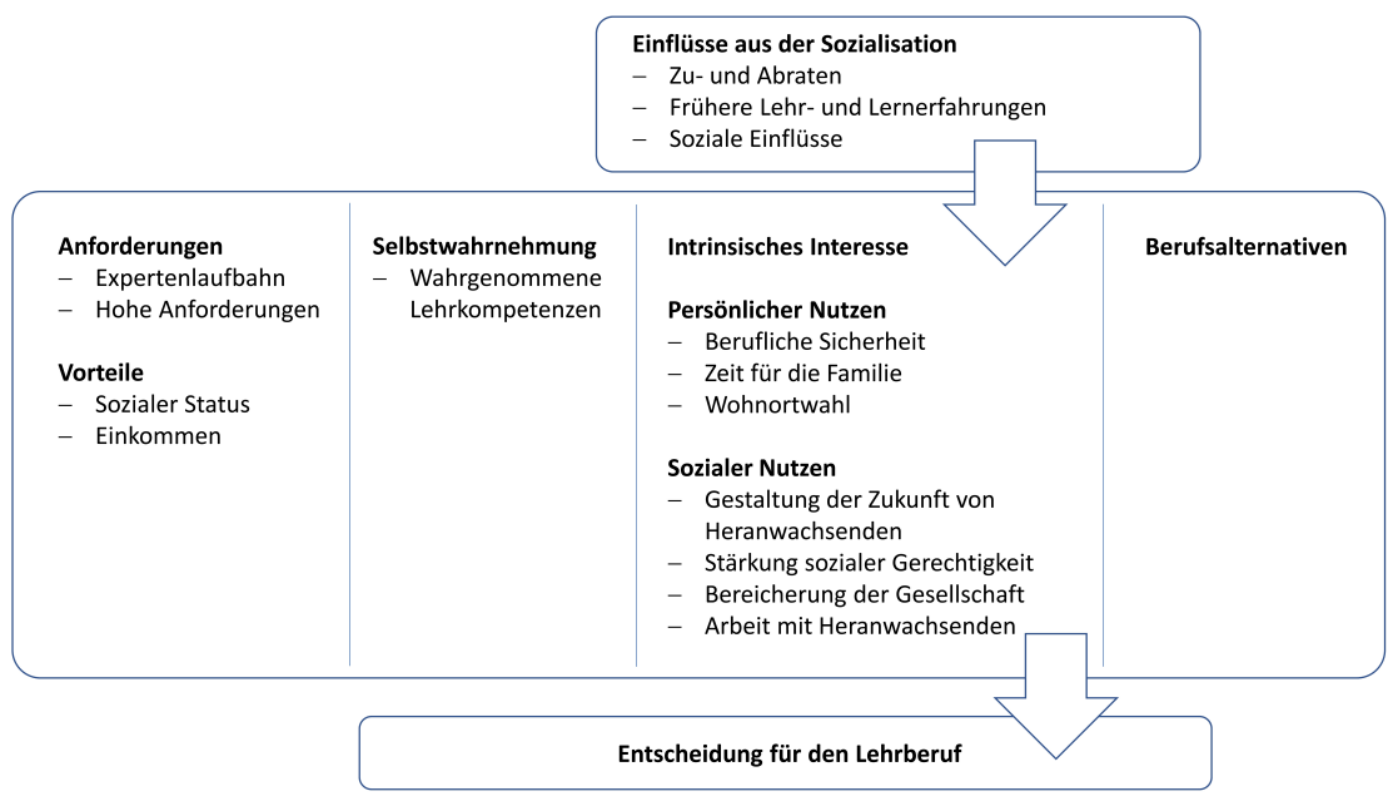

Abbildung 2. FIT-Choice Modell nach Watt und Richardson (2014; übersetzt durch den Autor)

Die wahrgenommenen Anforderungen und Vorteile des Lehrberufs entsprechen in ihrer Bilanz konzeptuell der Kosten-Komponente nach Eccles et al. (1983). Sie umfassen einerseits Einschätzungen, zu welchem Ausmaß spezialisierte und technische Kompetenzen Voraussetzungen für diesen Beruf sind und wie arbeitsintensiv und belastend dieser ist und andererseits, wie sehr der Lehrberuf respektiert und angesehen ist und wie gut die Verdienstmöglichkeiten sind. Selbstwahrgenommene Kompetenz beschreibt, wie sehr eine Person davon überzeugt ist, die notwendigen Kompetenzen für den Lehrberuf mitzubringen. Der subjektive Wert wurde vergleichbar zu Eccles et al. (1983) unterschieden und umfasst drei Faktoren, nämlich (a) das intrinsische Interesse am Unterrichten, (b) den persönlichen Nutzen des Lehrberufs für einen selbst, etwa hinsichtlich beruflicher Sicherheit, Zeit für die Familie oder die Wohnortwahl sowie (c) den wahrgenommenen sozialen Nutzen, beispielsweise inwieweit mit diesem Beruf die Zukunft von Heranwachsenden gestaltet werden kann und wie sehr er die Gesellschaft bereichert. Berufsalternativen beziehen sich schließlich darauf, welche Ausweichmöglichkeiten gegeben sind und ob der Lehrberuf als erste Wahl oder in Ermangelung an Alternativen verfolgt wird.

Die Abgrenzbarkeit dieser Berufswahlgründe wurde faktorenanalytisch, auch invariant über verschiedene Länder hinweg, bestätigt, sodass von einem methodisch-tragfähigen Zugang zur Analyse der Berufswahlmotivation von 
(angehenden) Lehrkräften ausgegangen werden kann (Watt \& Richardson, 2012). Darauf beruhende Studien erbrachten unter anderem, dass Berufsalternativen meist nur ein wenig bedeutsames Motiv darstellen, während selbstwahrgenommene Kompetenzen und der intrinsische Wert einen besonders hohen Stellenwert einnehmen - wobei typischerweise jedoch auch umfangreiche Anforderungen des Lehrberufs angenommen werden. Resultate von Studien aus verschiedenen Ländern ergänzten dieses Bild dahingehend, dass je nach kulturellem Kontext oder Schulsystem auch Unterschiede in der Bedeutsamkeit eher individualistischer oder kollektivistischer Motivationen vorliegen (Watt \& Richardson, 2012). Befunde zu den Auswirkungen dieser Gründe auf tatsächliche Unterschiede in der beruflichen Praxis gibt es bislang jedoch erst spärlich. Diese beruhen zudem meist auf querschnittlichen Untersuchungsdesigns, weswegen sie bedacht zu interpretieren sind, da Veränderungen oder Verzerrungen von Berufswahlgründen bei einer retrospektiven Erfassung möglich sind. Eine erste längsschnittliche Untersuchung von Richardson und Watt (2010) verweist darauf, dass Berufswahlgründe einen Einfluss auf das spätere, selbstberichtete Lehrverhalten haben. Besonders altruistische Gründe, ein hoher wahrgenommener sozialer Nutzen und selbsteingeschätzte Fähigkeiten führten zu einer günstigeren Selbsteinschätzung des Lehrhandelns nach bis zu acht Jahren, während soziale Beweggründe sowie eine Entscheidung aufgrund eingeschränkter Berufsalternativen abträglich waren. Günstige Motivkonstellationen erwiesen sich über die ersten fünf Jahre der Lehrtätigkeit hinweg als relativ stabil, führten jedoch auch zu geringerer Zufriedenheit und Persistenz (Richardson \& Watt, 2010). Dies impliziert, dass eine Anpassung der Berufsgründe notwendig sein mag, besonders wenn die berufliche Praxis in einem Kontext stattfindet, der das Erreichen der ursprünglichen Beweggründe nicht in vollem Umfang zulässt.

Zusammenfassend handelt es sich bei Berufswahlgründen somit um einen ertragreichen Zugang, um zu verstehen, welche Personen sich für den Lehrberuf entscheiden und inwieweit dies für Unterschiede in ihrem Handeln zu Beginn der Tätigkeit verantwortlich sein kann. Eine spannende Richtung ist aufbauend darauf zu eruieren, wie diese Berufswahlgründe auch für den tatsächlichen Verlauf oder ein vorzeitiges Ausscheiden aus dem Lehrberuf verantwortlich sein können (vgl. Richardson et al., 2014).

\section{Interesse}

Das pädagogisch-psychologische Konstrukt des Interesses (lat. inter-esse, dazwischen sein) ist durch seine Gegenstandsspezifität geprägt und als Relation zwischen Person und Gegenstand aufzufassen (Krapp, 2002; Prenzel \& Schiefele, 
2001). Eine Analyse von Interessensausprägungen impliziert also, dass sowohl personale als auch situationale Merkmale berücksichtigt werden müssen.

Je nachdem, wie stark diese bei der Betrachtung (theoretisch und empirisch) gewichtet werden, sind zwei verschiedene Zugänge zu unterscheiden (vgl. Krapp, 2001): einerseits ein strukturorientierter Ansatz, in dem dispositionale, über die Zeit stabile Orientierungen von Personen betrachtet werden. Dazu gehört beispielsweise der Ansatz von Holland (1996, 1997), der sechs basale Interessensorientierungen postuliert, die er als Teil der Persönlichkeit auffasst und auf Grundlage derer bestimmte Berufspräferenzen abgeleitet werden können.

Andererseits existiert ein prozessorientierter Ansatz. In dessen Rahmen betrachtet man jenes Interesse, das situational auftritt und eine laufende Interessenhandlung begleitet. Es kann entweder primär auf ein bereits bestehendes individuelles Interesse zurückgehen oder durch situations- und gegenstandsbezogene Anreize ausgelöst werden (Krapp \& Prenzel, 2011). Es hat eine affektive und eine kognitive Komponente (Renninger \& Hidi, 2002) - wobei Letztere eine wichtige Abgrenzung zu anderen Konstrukten, wie Enthusiasmus, darstellt (vgl. Kunter et al., 2008). Individuelles Interesse ist durch gerichtete Aufmerksamkeit, erhöhte kognitive Leistungsfähigkeit und emotionale Anteilnahme charakterisiert (Krapp, 2009) und geht einher mit dem Erleben von positiven emotionalen Zuständen während der Beschäftigung mit dem Interessensgegenstand, einer hohen subjektiven Wertschätzung dessen sowie dem Ziel, Wissen über den Gegenstand zu erweitern (Schiefele, 1974).

Das Konzept des Interesses wurde umfangreich bei Schüler(inne)n und Studierenden untersucht. Forschungsarbeiten erbrachten unter anderem, dass Interesse positiv mit Aufmerksamkeit, Lernqualität und Noten zusammenhängt (Ainley, Hidi \& Berndorff, 2002; Hidi \& Renninger, 2006; Schiefele, Krapp \& Winteler, 1992). Ein starkes individuelles Interesse hinsichtlich eines bestimmten Gegenstandes fördert - vermittelt über einen hohen intrinsischen Wert - die intensive Auseinandersetzung mit dem entsprechenden Gegenstand, was insbesondere das Erleben eigener Kompetenzen und somit eine positive Selbsteinschätzung eigener Fähigkeiten begünstigt und damit wiederum ebenso die Ausbildung und Stabilisierung des Interesses (vgl. Verortung im theoretischen Modell in Abbildung 1; s. a. Dresel \& Lämmle 2017; Wigfield, Eccles, Schiefele, Roeser \& Davis-Kean, 2006).

Aufbauend auf den umfangreichen Arbeiten zu Schüler(inne)n und Studierenden liegen erste Arbeiten vor, die das Konzept bei Lehrkräften untersuchen.

Lehrerinteresse wird dabei meist im Rahmen des jeweiligen Fachs aufgefasst und anhand von Merkmalen individuellen Interesses definiert (Eren, 2012; Long \& Woolfolk Hoy, 2006). Inhaltlich wird Interesse dabei meist weiter differenziert, 
indem das Interesse am Fach vom Interesse am Unterrichten unterschieden wird (Franz, 2008; Herranen, Vesterinen \& Aksela, 2015; Lange, 2010; Möller, 2004). Schiefele, Streblow und Retelsdorf (2013) schlugen vor, dass Interesse - ähnlich zu den Differenzierungen des pädagogischen Wissens von Lehrkräften in content knowledge, pedagogical content knowledge and general pedagogical knowledge (z. B. Baumert \& Kunter, 2006; Shulman, 1986) - in fachliches (z. B. für das Fach Mathematik), didaktisches (z. B. hinsichtlich Lehrmethoden zur Aufbereitung fachlicher Inhalte) und erzieherisches Interesse (z. B. hinsichtlich pädagogischen Aspekten und Fragen des Lehrberufs) untergliedert werden sollte. Anhand einer Stichprobe von 281 Lehrkräften unterschiedlicher Schulformen bestätigten sie diese dreidimensionale Struktur und lieferten Zusammenhänge mit dem beruflichen Erleben und dem selbstberichteten Instruktionsverhalten. Didaktisches und erzieherisches Interesse gingen mit geringerem Belastungserleben einher und standen im Zusammenhang mit günstigem Lehrverhalten wie etwa kognitiver Aktivierung. Fachliches und erzieherisches Interesse waren demgegenüber mit Vergnügen und Flow im Unterricht assoziiert. Zu ähnlichen Ergebnissen kam auch Eren (2012) mit türkischen Lehrkräften. Des Weiteren liegen bereits erste Zusammenhänge vor, die über den Unterrichtskontext hinausgehen: Rzejak und Lipowsky (2016) berichteten beispielsweise, dass besonders interessierte Lehrkräfte Lerngelegenheiten während Fortbildungen engagierter nutzten als weniger Interessierte.

Long und Woolfolk Hoy (2006) wählten einen anderen Forschungszugang: Sie befragten Schüler(innen) und fanden, dass diejenigen, die von großem Interesse ihrer Lehrkräfte berichteten, selbst auch sehr gute Noten hatten. Trotz Ermangelung an Forschungsbefunden, in denen Angaben von Lehrer(inne)n und Schüler(inne)n im engeren Sinne verknüpft werden, erscheint naheliegend, dass Lehrerinteresse auch mit Aspekten auf Schülerseite assoziiert ist. Trotz der relativ wenigen Forschungen zum Interesse von Lehrkräften verweist dies darauf, dass das Konzept des Lehrkraftinteresses einen wichtigen und eigenständigen Beitrag zur Erklärung von Unterschieden im Erleben und Verhalten der Lehrkräfte liefern kann (s. a. Reichhart, 2018). Gerade eine spezifische Erfassung des Interesses (bspw. auch hinsichtlich spezifischer Unterrichtsthemen) erscheint hierzu als eine spannende und vielversprechende Forschungsrichtung. Eine weitere interessante Perspektive bezieht sich auf die Entwicklung und Veränderung des Interesses, beginnend in der Lehrerausbildung. 


\section{Selbstwirksamkeitserwartungen}

Im Kern des Rahmenmodells (Abbildung 1) steht neben der Wünschbarkeit von Handlungsoptionen (Wert) deren Realisierbarkeit (Erwartung).

Erfolgserwartungen sind subjektive Einschätzungen einer Person darüber, mit welcher Wahrscheinlichkeit Erfolg bei Durchführung einer bestimmten Handlung eintritt. Dazu gehen neben der Erwartung darüber, mit welcher Wahrscheinlichkeit Erfolg durch eigenes Handeln herbeigeführt werden kann, auch Erwartungen darüber ein, wie sich die Situation entwickelt, wenn nicht eingegriffen wird. Gerade erstere, Handlungs-Ergebnis-Erwartungen (vgl. Heckhausen \& Rheinberg, 1980), werden als besonders bedeutsam für den Motivationsprozess erachtet (Überblick z. B. bei Schunk et al., 2013).

Eine entscheidende Grundlage entsprechender Erwartungen sind Selbstwirksamkeitserwartungen (Bandura, 1977, 1997, 2001): subjektive Annahmen einer Person darüber, ob sie selbst eine bestimmte Handlung erfolgreich ausführen kann; wobei die Frage, ob diese Handlung dann auch zu dem angestrebten Ergebnis führt, kein Bestandteil dieses Konzeptes ist. Aufgrund von Über- oder Unterschätzungen hängen Selbstwirksamkeitserwartungen nur bedingt mit den tatsächlichen Fähigkeiten einer Person zusammen (vgl. Schwarzer, 1996). Auch sollten Selbstwirksamkeitserwartungen klar von dem Selbstkonzept abgegrenzt werden. Letzteres bezeichnet - im Gegensatz zu den eher zukunftsbezogenen Selbstwirksamkeitserwartungen - meist aus der Vergangenheit gespeiste (Zimmerman \& Cleary, 2006), allgemeine Einschätzungen über die eigene Person, also beispielsweise Urteile, Einstellungen und Überzeugungen (Schunk \& Zimmerman, 2006). Somit ist das Selbstkonzept umfassender als die stärker aufgabenbezogenen Selbstwirksamkeitserwartungen und inkludiert diese, neben Kompetenzüberzeugungen und dem Selbstwertgefühl (Parajes, 1996).

Lehrkraft-Selbstwirksamkeitserwartungen beziehen sich darauf, wie sehr sich Lehrpersonen in der Lage sehen, durch eigenes Handeln pädagogisch herausfordernde Situationen zu bewältigen (vgl. Schmitz \& Schwarzer, 2000), d. h. das Lernen und Verhalten der Schüler(innen) zu unterstützen und zu fördern, gerade auch bei unmotivierten oder problematischen Schüler(inne)n (TschannenMoran \& Woolfolk Hoy, 1998, 2001). Wie auch für Schüler(innen), nehmen Selbstwirksamkeitserwartungen für Lehrkräfte eine wichtige Rolle für ihr Handeln und Leisten ein (Ross, 1992). Es handelt sich dabei um ein generisch motivationspsychologisches Konstrukt, das bereits recht früh und sehr produktiv in die Lehrkraftforschung eingeflossen ist (Klassen, Durksen \& Tze, 2014).

Erste Forschungen zu Lehrkraft-Selbstwirksamkeitserwartungen gehen auf Gibson und Dembo (1984) zurück, waren jedoch die ersten zwanzig Jahre von relativ 
unsystematischen Foki und Messinstrumenten geprägt und bildeten oft im Kern nicht das ursprüngliche Selbstwirksamkeits-Konstrukt sensu Bandura ab, bis sich einheitlichere Konzeptualisierungen und Zugänge durchsetzten (Tschannen-Moran \& Woolfolk Hoy, 2001). Aktuell ist die Konzeptualisierung sensu TschannenMoran und Woolfolk Hoy höchstwahrscheinlich der meist gebrauchteste Zugang zur Lehrkraft-Selbstwirksamkeitserwartung (Klassen, Tze, Betts \& Gordon, 2011).

\section{Spezifität und Dimensionalität}

Es gibt unterschiedliche Spezifitätsgrade, wie Selbstwirksamkeitserwartungen aufgefasst und gemessen werden sollten (vgl. Tschannen-Moran \& Woolfolk Hoy, 2001). Grundsätzlich kann neben einer allgemeinen Selbstwirksamkeitserwartung (die alle Lebensbereiche umfasst und eine generelle Lebensbewältigungskompetenz ausdrückt) eine situationsspezifische Selbstwirksamkeitserwartung sowie eine bereichsspezifische Selbstwirksamkeitserwartung, die hinsichtlich ihrer Spezifität zwischen allgemeiner und situationsspezifischer Selbstwirksamkeitserwartung liegt, unterschieden werden (zsf. Warner \& Schwarzer, 2009). Da sich LehrkraftSelbstwirksamkeitserwartungen auf Anforderungen des Berufslebens beziehen, nehmen diese den Rang einer bereichsspezifischen Selbstwirksamkeitserwartung ein (Warner \& Schwarzer, 2009). Sie kann jedoch je nach Forschungsfrage weiter in spezifische Selbstwirksamkeitsformen untergliedert werden, z. B. hinsichtlich des Classroom-Managements, der Motivierung von Schüler(inne)n, der Kooperation mit Eltern oder der Vermittlung von Inhalten in einem bestimmten Fach (z. B. Skaalvik \& Skaalvik, 2007; Tschannen-Moran, Woolfolk Hoy \& Hoy, 1998; Wolters \& Daughtery, 2007).

Abgesehen davon gibt es neben individuellen Selbstwirksamkeitserwartungen auch kollektive Selbstwirksamkeitserwartungen. Dabei handelt es sich um Einschätzungen hinsichtlich der Wirksamkeit als Gruppe, die sich aus der Koordination und Kombination der verschiedenen Beteiligten ergibt (Bandura, 1997; Myers \& Feltz, 2007; Schwarzer \& Schmitz, 1999). Analog zu individuellen Selbstwirksamkeitserwartungen beschreiben kollektive Selbstwirksamkeitserwartungen das Vertrauen einer Gruppe in deren Handlungskompetenz (vgl. Bandura, 1997). Für Lehrkräfte ist die relevanteste Gruppenebene das Lehrerkollegium, wobei entsprechende kollektive Selbstwirksamkeitserwartungen Einfluss darauf haben, wie gut das soziale System Schule funktioniert (vgl. Bandura, 1993). Allerdings liegen bislang nur wenige empirische Untersuchungen zu diesem Konstrukt vor, da sich die meisten Forschungsarbeiten auf individuelle Selbstwirksamkeitserwartungen beziehen, die, sofern nicht anders spezifiziert, im Folgenden gemeint sind. 


\section{Zusammenhänge mit Lehrkraft-Gesundheit, Lehrhandeln und Schülermerkmalen}

Forschungsarbeiten zu den Folgen von Lehrkraft-Selbstwirksamkeitserwartungen untersuchten häufig Zusammenhänge mit der Gesundheit der Lehrkräfte (z. B. Edgyed \& Short, 2006; Schwerdtfeger, Konermann \& Schönhofen, 2008; Skaalvik \& Skaalvik, 2007; vgl. Review von Brown 2012). Schmitz und Schwarzer (2002) berichteten beispielsweise mittlere bis hohe Zusammenhänge individueller und kollektiver Lehrer-Selbstwirksamkeitserwartung mit den im Maslach Burnout Inventory unterschiedenen Facetten des Belastungserlebens (emotionaler Erschöpfung, Depersonalisierung und Leistungsverlust; Maslach, Jackson \& Leiter, 1996) über eine Zeitdistanz von ein oder zwei Jahren hinweg, wobei Cross-Lagged-Panel Analysen nahelegten, dass die

Selbstwirksamkeitserwartung eine protektive Rolle einnimmt (Schwarzer \& Hallum, 2008). Auch Abele und Candova (2007) berichten günstige Einflüsse hoher Selbstwirksamkeitserwartungen auf das Belastungserleben über einen längeren Zeitraum. Darüber hinaus werden Zusammenhänge mit verwandten Konstrukten, wie etwa der Berufszufriedenheit, berichtet (z. B. Betoret, 2006; Klassen \& Chiu, 2010; Wolters \& Daugherty, 2007) und es gibt erste Erkenntnisse, dass Selbstwirksamkeitserwartungen nicht nur mit dem (selbsteingeschätzten) Belastungserleben, sondern auch mit körperlichen Parametern (wie Cortisol-Werten, Veränderungen der Herzfrequenz im Unterricht, körperlichen Beschwerden; auch unter Kontrolle des allgemeinen Lebensstils sowie demographischer Angaben) assoziiert sind (Schwerdtfeger et al., 2008).

Neben der Gesundheit von Lehrpersonen widmeten sich viele Forschungsarbeiten zu diesem Konstrukt dem (oft durch die Lehrkräfte selbsteingeschätzten) Lehrhandeln. Es wird berichtet, dass Lehrkräfte mit hohen Selbstwirksamkeitserwartungen, im Vergleich zu Kolleg(inn)en mit niedrigeren Selbstwirksamkeitserwartungen, günstigeres instruktionales Handeln aufweisen, sich also etwa mehr um Schüler mit Lernschwierigkeiten kümmern, innovativer und reflektierter lehren, häufiger aktivierende Unterrichtstechniken anwenden und ein günstigeres Klassenklima erreichen (zsf. Klassen et al., 2014; Schunk et al., 2013; Schwarzer \& Warner, 2014). Außerdem wird berichtet, dass Selbstwirksamkeitserwartungen ein wichtiger Prädiktor für das Engagement von Lehrkräften, auch außerhalb des Unterrichts, sind (Coladarci, 1992; Schmitz \& Schwarzer, 2002) und Lehrkräfte mit hohen Selbstwirksamkeitserwartungen ihren Schüler(inne)n auch höhere Lernziele setzen (vgl. Wolters \& Daughterty, 2007). Klassen und Tze (2014) bekräftigten entsprechende Befunde, dass Lehrkräfte mit hoher Selbstwirksamkeitserwartung eine bessere Lehrqualität haben, indem sie die wenigen Arbeiten, in denen fremdeingeschätzte Lehrqualitätsaspekte erfasst 
wurden, meta-analytisch betrachteten und eine kleine, aber statistisch signifikante Effektgröße von $r=.28$ fanden.

Darüber hinaus wurden sowohl für individuelle als auch für kollektive LehrkraftSelbstwirksamkeitserwartungen erste Zusammenhänge mit den Leistungen der unterrichteten Schüler(innen) berichtet (Ashton \& Webb, 1986; Caprara et al., 2006; Ross, 1992; Tschannen-Moran \& Barr, 2004; auch die Meta-Analyse von Klassen und Tze, 2014, lieferte hierzu einen recht kleinen, aber statistisch signifikanten, Effekt). Ferner gibt es erste Erkenntnisse, dass die Selbstwirksamkeitserwartungen der Lehrkräfte auch mit motivationalen Merkmalen ihrer Schüler(innen) zusammenhängen: Berichten Lehrkräfte über eine hohe Selbstwirksamkeitserwartung, so sind die Überzeugen der Schüler(innen) hinsichtlich ihrer eigenen Leistungen und ihrem Potential größer und sie schätzen Schwierigkeiten im jeweiligen Unterrichtsfach als geringer ein (Klassen et al., 2011; Midgley, Feldlaufer \& Eccles, 1989; Ross, Hogaboam-Gray \& Hannay, 2001; Stipek et al., 2001).

\section{Veränderbarkeit und zeitliche Entwicklung}

Theoretisch ist anzunehmen, dass Selbstwirksamkeitserwartungen, wenn sie einmal entwickelt sind, überwiegend stabil sind. Empirisch existieren allerdings bislang noch kaum Erkenntnisse zu ihren Veränderungen über die Zeit als Lehrkraft hinweg (Klassen et al., 2014). Erste Erkenntnisse zu frühen Lehramtsstadien sowie zur Lehrerausbildung im Praktikum verweisen darauf, dass sich Selbstwirksamkeitserwartungen durchaus verändern und angepasst werden, gerade auch in kurzen, intensiven Perioden und bei Übergängen (z. B. dem „Praxisschock“; Klassen \& Durksen, 2014; Woolfolk Hoy \& Spero, 2005). Über die gesamte berufliche Tätigkeit hinweg liegen bislang keine längsschnittlichen Studien vor. Querschnittliche Befragungen, in denen Lehrkräfte in verschiedenen Stadien ihrer beruflichen Laufbahn vertreten waren, verweisen jedoch auf eine mögliche Entwicklung der Selbstwirksamkeitserwartungen wie bei einer invertierten U-Kurve: Bis zum Ende des mittleren Karriereabschnitts (ca. die ersten 23 Jahre) lagen bei Klassen und Chiu (2010, 2011) steigende, danach wieder abfallende Selbstwirksamkeitserwartungen vor.

Welche Prozesse können solche Veränderungen der Selbstwirksamkeitserwartungen erklären? Zur Entstehung und Beeinflussung von Selbstwirksamkeitserwartungen verwies Bandura auf die Bedeutung von (a) Kompetenzerwartungen aus eigenen, nachhaltigen Erfolgserfahrungen, (b) stellvertretenden Erfahrungen durch Beobachtung von Verhaltensmodellen, (c) verbalen Überzeugungen sowie (d) Wahrnehmungen eigener physischer Erregung (Bandura 1977, 1997). Bei Lehrkräften wird angenommen, dass die gleichen 
Quellen zutreffen (vgl. Schwarzer \& Warner, 2014). Beispielsweise wurde berichtet, dass sich verbale Überzeugungen in Form von Ermutigungen bei unerfahrenen Lehrkräften auf ihre Selbstwirksamkeitserwartungen auswirken (vgl. Hagen et al., 1998; Tschannen-Moran \& Woolfolk Hoy, 2007). Außerdem wird berichtet, dass Modelllernen (beispielsweise mit Hilfe eines erfahrenen Kollegen oder anhand von Videos) eine sinnvolle Strategie zur Einführung neuer Lehrmethoden ist und dass es sich anbietet, Erfahrungen mit neuen Methoden in einem offenen, unterstützenden Kollegenkreis zu diskutieren (z. B. Hagen et al., 1998; Schunk \& Hanson, 1985; Zimmerman \& Kitsantas, 2002). Erste experimentelle Untersuchungen bestätigen dies und verweisen auf die gezielte Veränderbarkeit der Selbstwirksamkeitserwartungen: O’Connor und Korr (1996) berichteten eine günstigere Entwicklung der Selbstwirksamkeitserwartungen in einer Gruppe von Lehrkräften, die Peer-Coaching durchführten, als in einer Kontrollgruppe, die ihren Unterricht ohne Beobachtung und Feedback in kollegialer Atmosphäre durchführte. Darüber hinaus liegen erste Indizien vor, dass Veränderungen der Lehrkraft-Selbstwirksamkeitserwartungen auch durch Interventionen auf Gruppen- (Posnanski, 2002; Ross \& Bruce, 2007; Wudy \& Jerusalem, 2011) oder Schulebene (Kelm \& McIntosh, 2012) möglich sind.

\section{Fazit zu Selbstwirksamkeitserwartungen von Lehrkräften}

Zusammenfassend lassen sich somit bereits umfangreiche empirische Beschäftigungen mit dem Konstrukt der Lehrkraft-Selbstwirksamkeitserwartungen ausmachen, die sich seit der Jahrtausendwende hinsichtlich Konzeptions- und Messzugängen deutlich vereinheitlicht haben. Dennoch fordern Klassen und Kolleg(inn)en (2011) in einem Überblick zum Forschungsstand der LehrerSelbstwirksamkeitserwartungen von 1998 bis 2009 noch mehr Bemühungen um valide Skalen zur Erfassung der Selbstwirksamkeitserwartung. Zum besseren Verständnis der Selbstwirksamkeitserwartungen und für einen Vergleich von Forschungsarbeiten scheint es außerdem hilfreich, das Spezifitätsniveau stets klar festzulegen und zu kommunizieren.

Während die Zusammenhänge mit Konstrukten auf Lehrerseite selbst als recht gut belegt erachtet werden können, wird gefordert, dass mehr Forschung zu den Zusammenhängen mit (vor allem extern gemessenen) Resultaten auf Schülerebene notwendig ist (Klassen et al., 2014). Entsprechende Forschungsbemühungen sollten darüber hinaus auch vermittelnde Mechanismen in den Blick nehmen, über die bislang noch wenig bekannt ist. 


\section{Zielorientierungstheorie}

Die in Abschnitt 2 beschriebenen Definitionen von Motivation (als aktivierende Ausrichtung auf positiv bewertete Zielzustände) verweisen darauf, dass Ziele eine bedeutende Rolle im motivierten Handeln spielen (Dresel \& Lämmle, 2017).

Ziele sind Vorwegnahmen von Handlungsfolgen, die mehr oder weniger bewusst zustande kommen und sich auf zukünftige, angestrebte Handlungsergebnisse sowie deren kognitive Repräsentationen beziehen (Kleinbeck, 2010). Sie erfüllen im psychischen System mehrere Funktionen: Unter anderem veranlassen sie auf das im Ziel enthaltene Resultat ausgerichtete Handlungen, strukturieren den Einsatz von Wissen und Fähigkeiten im Handeln und stellen einen Maßstab zur Beurteilung entsprechender Handlungen und deren Abschluss dar. Durch die Beschreibung von Zielen kann entsprechend die Wert-Komponente für verschiedene Handlung(soption)en relativ breit konzipiert werden.

Die Zielorientierungstheorie beschreibt in Form von vergleichsweise überdauernde motivationale Tendenzen, welche Arten von Zielen Personen in sozialen Lern- und Leistungskontexten bevorzugt verfolgen (z. B. Dweck, 1986; Elliot \& Hulleman, 2017; Nicholls, 1984). Unterschiedliche, auf die eigene Person bezogene Ziele können hinsichtlich ihrer Charakteristika und Fokussierungen als Zielklassen konsolidiert werden. Als solche repräsentieren sie ein strukturiertes theoretisches System beziehungsweise Schema zur Initiierung, Durchführung und Evaluation von Handlungsweisen. Von diesem wird angenommen, dass es sich unter anderem auf die Verarbeitung selbst- und aufgabenbezogener Informationen, Bewertungsmaßstäbe, Schlussfolgerungen auf eigene Fähigkeiten und kausale Überzeugungen und somit auf Affekt, Kognition und Verhalten in Lern- und Leistungssituationen auswirkt (Elliott \& Dweck, 1988).

Individuelle Präferenzen für bestimmte Arten von Zielen wurden ursprünglich als „goal orientations“ (Zielorientierungen) bezeichnet. Dieser Begriff suggeriert jedoch (fälschlicherweise), dass primär eine bestimmte Klasse von Zielen verfolgt wird (ein aktuelles Verständnis geht davon aus, dass mehrere Arten von Zielen simultan, im Sinne multipler Zielsetzungen, verfolgt werden; Barron \& Harackiewicz, 2001; Pintrich, 2000b). Auch impliziert diese Bezeichnung nomenklatorisch einen recht dispositionellen Charakter, der auf zugrundeliegende Handlungsgründe fokussiert ist, jedoch im Widerspruch zu aktuellen Forschungsbefunden zur Stabilität der Ziele und einem theoretischen Fokus auf Handlungszwecke steht. Im Englischen wurde dem Rechnung getragen, indem stattdessen der Terminus "achievement goals" verwendet wird. In der deutschen Literatur existiert jedoch, trotz ebenfalls veränderter Definitionen und Operationalisierungen, kein etabliertes sprachliches Pendant zu diesem Begriff. 
Daher wird hier von Präferenzen für selbstbezogene Ziele gesprochen, und als synonyme, jedoch assoziativ neutrale Alternative zum Zielorientierungsbegriff präferiert (vgl. Daumiller, 2018). Insbesondere erscheint diese Bezeichnung sehr tragfähig zur sprachlich präzisen Charakterisierung der Motivation von Lehrenden, die häufig auch Ziele haben, die sich nicht auf die eigene Person richten (z. B. didaktische Lehr-Ziele) und nicht in der Zielorientierungstheorie gemeint sind.

Die Zielorientierungstheorie ist ein etablierter theoretischer Rahmen zur Erklärung und Vorhersage von Lern- und Leistungshandeln unterschiedlicher Populationen (Überblicke bei Elliot \& Hulleman, 2017; Maehr \& Zusho, 2009; Meece, Anderman \& Anderman, 2006) und wurde aufbauend auf umfangreicher Forschungsliteratur zu Schüler(inne)n und Studierenden in jüngster Zeit erfolgreich auf Lehrkräfte und ihr berufliches Handeln übertragen (Butler, 2007). Die zugrundeliegende Prämisse dafür ist, dass sich Lehrkräfte in Schule und Unterricht ebenso wie ihre Schüler(innen) in einem Lern- und Leistungskontext befinden und oft genauso danach streben können, ihre eigenen Kompetenzen zu demonstrieren, Defizite zu verbergen und ihr eigenes professionelles Wissen und Können zu erweitern. Zahlreiche empirische Befunde aus unterschiedlichen Ländern bestärken mittlerweile, dass Ziele von Lehrkräften mit ihrem Wohlbefinden, professionellem Lernverhalten und Unterrichtshandeln zusammenhängen (Überblicke z. B. bei Butler, 2014; Nitsche et al., 2017).

\section{Struktur der Ziele}

Hinsichtlich der verschiedenen Arten selbstbezogener Ziele wird primär zwischen Lernzielen (Fokus auf Erweiterung eigener Kompetenz) und Performanzzielen ${ }^{1}$ (Fokus auf eigene Kompetenz im sozialen Vergleich und wie diese von anderen wahrgenommen wird) unterschieden. Zusätzlich gibt es eine Unterscheidung zwischen Annäherungs- (Streben nach dem Erreichen positiver Zustände) und Vermeidungszielen (Streben nach der Vermeidung negativer Zustände; Elliot \& Harackiewicz, 1996; Elliot \& McGregor, 2001; Middleton \& Midgley, 1997).

In den Arbeiten zu Lehrkräften werden primär fünf Klassen von Zielen unterschieden: Eine Lehrkraft verfolgt ein (Annäherungs-)Lernziel, wenn sie bestrebt ist, selbst etwas dazuzulernen und ihre professionellen Kompetenzen zu

\footnotetext{
${ }^{1}$ Im Deutschen ist auch die Bezeichnung Leistungsziele geläufig. Aufgrund der begrifflichen Überlappung mit dem Begriff der Leistungsmotivation wird zur Vermeidung von Missverständnissen hier der Performanz-Begriff präferiert.
} 
erweitern, etwa indem neue Ansätze im Unterricht ausprobiert oder Fortbildungen besucht werden. Annäherungsperformanzziele beschreiben ein Bestreben danach, besser als andere zu sein und einen guten Eindruck zu machen, beispielsweise gegenüber Kolleg(inn)en, Schüler(inne)n oder Vorgesetzten (vgl. Nitsche, Dickhäuser, Fasching \& Dresel, 2011). Demgegenüber verfolgt eine Lehrkraft Vermeidungsperformanzziele, wenn es ihr darum geht, nicht schlechter als andere zu sein und einen (vermeintlichen) Kompetenzmangel zu verbergen. Daneben werden auch Arbeitsvermeidungsziele (Nicholls, 1984) betrachtet. Diese beschreiben ein Bestreben danach, einen möglichst geringen Arbeitsaufwand zu haben, z. B. den Unterricht möglichst effizient vor- und nachzubereiten. Abgesehen davon wurden in der Forschungsliteratur zum Zielstreben von Lehrkräften auch Beziehungsziele als weitere relevante Zielklasse postuliert (Butler, 2012). Entsprechende Ziele sind darauf gerichtet, eine freundschaftliche und wertschätzende Beziehung mit Schüler(inne)n aufzubauen.

Darüber hinaus existieren differenziertere Modelle zur Struktur der Ziele von Lehrenden (vgl. Abbildung 3 für eine Übersicht unterschiedener Zielklassen) in denen vorgeschlagen wird, hinsichtlich der Lernziele zu unterscheiden, ob absolute Standards (Aufgabenziele) oder intrapersonale Standards (Lernziele im engeren Sinne) zur Beurteilung der Zielerreichung angelegt werden sowie dass auch Vermeidungsaspekte bei diesen Zielklassen (beispielsweise das Bestreben, bereits vorhandene fachliche Kompetenzen nicht zu vergessen) relevant sein könnten (Daumiller, Dickhäuser \& Dresel, 2018; Mascret, Elliot \& Cury, 2015). Zusätzlich wird argumentiert, dass auch Performanzziele klar dahingehend getrennt werden sollten, ob ein Fokus auf dem normativen Abschneiden (z. B. besser als andere zu sein; Normziele) oder auf der Erscheinung liegt (z. B. einen guten Eindruck machen, unabhängig davon, wie kompetent man tatsächlich ist; Erscheinungsziele) - denn diese unterschiedlichen Aspekte können differenziell mit unterschiedlichem Erleben und Verhalten assoziiert sein (Daumiller et al., 2018; Daumiller, Grassinger, Dickhäuser \& Dresel, 2016; s. a. Hulleman et al., 2010; Senko \& Dawson, 2017). Zukünftige Forschungsarbeiten sollten entsprechenden Unterschieden der Lern- und Performanzziele - je nachdem, welche Facette betrachtet wird - weiter nachgehen, um ein umfassendes Verständnis des Zielstrebens von Lehrenden und seinen Auswirkungen zu erreichen. In jedem Fall sollte jedoch eine präzise und klare Bezeichnung der untersuchten Zielklassen erfolgen, um dadurch systematische und präzise Operationalisierungen und ein adäquates Einordnen verschiedener Forschungsbefunde zu ermöglichen. 


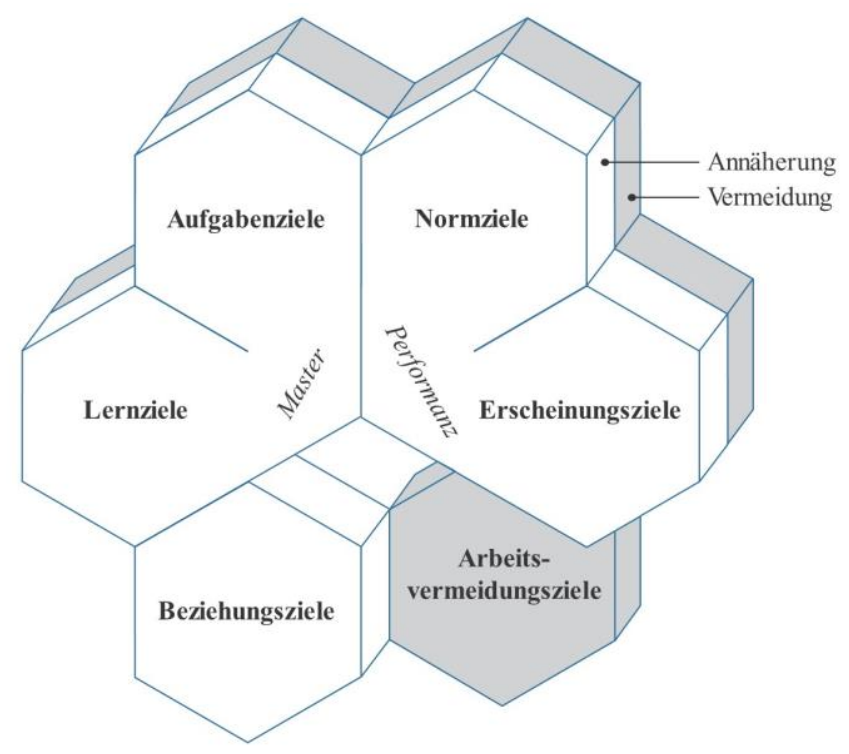

Abbildung 3. Übersichtsmodell zu den verschiedenen Klassen selbstbezogener Ziele im Lehrkontext nach Daumiller et al. (2018)

\section{Zusammenhänge mit Kognition, Handeln und Affekt}

Eine Reihe an empirischen Studien lieferte Hinweise dafür, dass das Wohlbefinden und die Selbstregulation von Lehrkräften von deren selbstbezogenen Zielen abhängen (Überblicke bei Butler, 2014 und Daumiller, 2018). Als Risikofaktor wurde vor allem eine starke Präferenz für Vermeidungsperformanzziele identifiziert: Lehrkräfte, die danach streben, eigene - vermeintliche - Inkompetenz zu verbergen und nicht schlechter bewertet zu werden als Kolleg(inn)en, berichten typischerweise auch eine geringere Zufriedenheit und geringeres Wohlbefinden im Beruf sowie geringeres Engagement, mehr Prokrastination und stärkere emotionale Erschöpfung (Nitsche et al., 2011; Papaioannou \& Christodoulidis, 2007; Parker et al., 2012; Retelsdorf et al., 2010; Tönjes \& Dickhäuser, 2009). Demgegenüber gilt das Verfolgen von Annäherungslernzielen als Schutzfaktor: Eine Präferenz für diese Zielklasse ging in den angeführten Studien mit größerer Berufszufriedenheit, adaptiveren Bewältigungsstrategien und geringerer emotionaler Erschöpfung einher.

Abgesehen davon wird berichtet, dass eine ausgeprägte Präferenz für Lernziele mit der Wahrnehmung von Hilfe als nützlich, dem Einholen von Feedback, dem Besuch von Fortbildungen, vermehrter Lektüre von Fachzeitschriften sowie einer besseren Leistung im Referendariat einhergeht (Butler, 2007; Dickhäuser et al., 
2007; Fasching et al., 2010; Nitsche et al., 2011, 2013; Runhaar et al., 2010).

Demgegenüber legen diese Studien nahe, dass starke (v.a.

Vermeidungs-)Performanzziele sowie Arbeitsvermeidungsziele damit

einhergehen, dass Lehrkräfte das Einholen von Hilfe als bedrohlich wahrnehmen, mit geringerer Wahrscheinlichkeit an Fortbildungen teilnehmen und im Referendariat schlechtere Leistungen sowie eine erhöhte Abbruchneigung zeigen.

Schließlich werden ebenfalls Zusammenhänge mit dem Unterrichtshandeln selbst berichtet. Lehrkräfte mit einer stärkeren Präferenz für Annäherungslernziele verwenden häufiger Instruktionsstrategien, die Schüler(innen) kognitiv stimulieren, bieten Schüler(inne)n mehr Unterstützung an und bewerten Leistungen häufiger nach individueller statt sozialer Bezugsnorm; darüber hinaus realisieren sie insgesamt in stärkerem Maße einen Unterricht, in dem das Lernen und der Erwerb von Kompetenzen - also schülerbezogene Lernziele - ins Zentrum gerückt werden (Butler \& Shibaz, 2008; Dresel et al., 2013; Han et al., 2015, 2016; Retelsdorf et al., 2010; Retelsdorf \& Günther, 2011; Shim et al., 2013; Thoonen et al., 2011; Wang et al., 2017). Umgekehrt fanden sich Hinweise, dass Lehrkräfte mit starken Vermeidungsperformanzzielen (für Annäherungsperformanzziele fanden sich inkonsistente Befundmuster) häufiger Instruktionsstrategien nutzen, die auf Wettbewerb und Konkurrenz ausgerichtet sind, Leistungen verstärkt nach der sozialen Bezugsnorm beurteilen, Fragen von Schüler(inne)n eher verhindern und deren Leistung ins Zentrum des Unterrichtsgeschehens rücken. Darüber hinaus liegen erste Befunde dafür vor, dass sich diese Effekte auch in der Motivation von Schüler(inne)n fortsetzen: So fanden Dresel, Fasching, Steuer, Dickhäuser und Daumiller (2018), dass günstige Lehrkraft-Zielpräferenzen mit günstigen Zielpräferenzen ihrer Schüler(innen) einhergingen und dass diese Effekte über die im Unterricht saliente Zielstruktur vermittelt waren (vgl. Ames 1992; Meece et al., 2006).

\section{Stabilität und Veränderbarkeit der Lehrkraft-Ziele}

Abseits der Auswirkungen des Zielstrebens von Lehrkräften verweisen erste Studien auf dessen grundsätzliche Beeinflussbarkeit und untersuchten Bedingungsfaktoren davon (z. B. Janke et al., 2015; Praetorius et al., 2014). Diese Arbeiten indizierten, dass das Zielstreben von Lehrkräften stabile sowie variable Anteile hat, von denen angenommen werden kann, dass sie auf stabile Charakteristika der Person (z. B. Motive) oder des Kontexts (z. B. Evaluationskultur) sowie auf variable Merkmale der Person (z. B. aktuelles Interesse) oder des Kontexts (z. B. Lerngelegenheit) zurückgeführt werden können (Praetorius et al., 2014). Dies fügt sich unmittelbar in die Abgrenzung aktueller 
Motivation und motivationaler Tendenzen im vorgeschlagenen Rahmenmodell der Lehrkraftmotivation in Abbildung 1 ein.

Aufbauend auf der unterschiedlichen Funktionalität der verschiedenen Präferenzen selbstbezogener Ziele und deren prinzipieller Veränderbarkeit widmete sich die Forschung zu Lehrkraftzielen möglichen Maßnahmen zur Unterstützung und Förderung günstiger Ziele. Diese Forschungsrichtung fußt unter anderem auf den Arbeiten zur Veränderung von Präferenzen von Zielen bei Schüler(inne)n und Studierenden, in denen grundlegend zwei Beeinflussungsmechanismen unterschieden wurden (vgl. Elliot \& Hulleman, 2017): einerseits die umweltzentrierte Gestaltung des sozialen Lern- und Leistungskontexts, sodass bestimmte Ziele salient und von den betreffenden Personen übernommen werden (vgl. dazu Abschnitt 8 für entsprechende Erkenntnisse zu Schullehrkräften); andererseits die direkte und personenzentrierte Beeinflussung der Ziele der betreffenden Personen (z. B. Bernacki et al., 2016; Hoyert \& O’Dell, 2006; Noordzij et al., 2013). In einer ersten Arbeit wurde versucht, selbstbezogene Ziele von Lehrkräften direkt zu beeinflussen: Benning et al. (2018) entwickelten einen Interventionsansatz zur Förderung der Lernzielorientierung bei Lehrkräften. An drei Workshop-Tagen wurde Lehrkräften Wissen zu Zielorientierungen vermittelt, dessen Implementierung sie, gestützt durch Feedback, in praktischen Phasen einübten und anhand eines Tagebuchansatzes reflektierten. Eine Evaluation dieses Ansatzes mit 40 Lehrkräften und einer Kontrollgruppe mit 29 Lehrkräften indizierte einen hohen subjektiven Lernertrag und wahrgenommenen Nutzen seitens der Lehrkräfte sowie eine sehr große Zufriedenheit. Es konnten jedoch noch keine zeitlichen Veränderungen der Lehrkraftziele nachgewiesen werden. Dies verweist somit auf einen vielversprechenden Ansatz, jedoch auch auf die Notwendigkeit von mehr Forschungsarbeiten in dieser Richtung, um die Erkenntnisse zur Funktionalität einzelner Zielklassen zur direkten Unterstützung des Zielstrebens von Lehrkräften in ihrer beruflichen Praxis anwenden zu können.

\section{Fazit zur Zielorientierungstheorie bei Lehrkräften}

Somit kann festgehalten werden, dass Lehrkräfte eine Vielzahl selbstbezogener Ziele verfolgen, die relevant für affektive, kognitive und behaviorale Aspekte im und über den Unterricht hinaus sind und zudem für die Motivation ihrer Schüler(innen) bedeutsam erscheinen. Verschiedene Zielklassen sind dabei unterschiedlich funktional - eindeutig günstige Zusammenhänge finden sich für eine Präferenz an Annäherungslernzielen, während eine Präferenz von Vermeidungsperformanzzielen und Arbeitsvermeidungszielen ungünstige Effekte hat (vgl. Maehr \& Zusho, 2009). Gerade für Annäherungsperformanzziele ist weitere Forschung notwendig, um besser zu verstehen, in Bezug auf welche 
Prozesse (oder für welche Personen) diese günstige oder ungünstige Effekte haben. Zur Aufklärung der bislang inkonsistenten Befundmuster scheint wichtig, diese Zielklassen trennscharf zu betrachten, etwa indem klar definiert und gemessen wird, ob ein Fokus auf Erscheinungs- oder Normaspekten liegt (die für jeweils unterschiedliches Erleben und Verhalten relevant sein könnten; vgl. Daumiller, 2018). Gerade in Anbetracht der Vielzahl an Zielklassen sind darüber hinaus ggf. auch personenzentrierte Ansätze (z. B. Zielprofile) gewinnbringend, die bislang noch kaum in der Forschung zu Lehrer-Zielen Einsatz finden (Kunst, van Woerkom \& Poell, 2018). Künftige Forschungsarbeiten würden außerdem davon profitieren, stärker Aspekte auf Schülerseite als abhängige Variablen zu betrachten und weiter zu explorieren, wie günstige Lehrkraftziele gefördert werden können.

\section{Zielstruktur}

Neben der personenzentrierten Beeinflussung von Zielpräferenzen wird in der Zielorientierungstheorie auch die umweltzentrierte Gestaltung des sozialen Lernund Leistungskontexts thematisiert. Durch diesen können Ziele salient und von den betreffenden Personen übernommen werden (vgl. Elliot \& Hulleman, 2017).

Zu den Effekten des (Lern- und Leistungs-)Kontexts auf individuelle Zielpräferenzen wird die Zielstruktur und deren Wahrnehmung durch die Individuen betrachtet (Überblick bei Meece et al., 2006). Die saliente Zielstruktur wird dabei als das Ausmaß definiert, in dem der Kontext das Verfolgen von selbstbezogenen Lern- oder Performanzzielen vorgibt oder ermöglicht. Im Unterrichtskontext wurde dazu für Schüler(innen) das Konzept der (Klassen-)Zielstruktur intensiv untersucht. Eine Vielzahl an empirischen Arbeiten indizierte, dass eine wahrgenommene Lernzielstruktur mit adaptiven Einstellungen, Gefühlen, Beziehungen, Lernstrategien und Persistenz seitens der Schüler(innen) in Beziehung steht - im Gegensatz zu einer Performanzzielstruktur, für die sich ungünstige Zusammenhänge finden (vgl. Skaalvik \& Skaalvik, 2017, für einen Überblick). Außerdem steht die wahrgenommene Zielstruktur wie erwartet in Korrespondenz zu den entsprechenden Zielen der Schüler(innen) (Kaplan \& Maehr, 1999; Urdan \& Midgley, 2003). Genauso wie Schüler(innen) erhalten auch Lehrkräfte Signale, welche Ziele und Werte an der Schule besonders wertgeschätzt und betont werden (Skaalvik \& Skaalvik, 2011, 2013). Eine entsprechende Schulzielstruktur bezieht sich auf die Erwartungen, Praktiken und Ziele einer Schule, die sich darauf auswirken, welche Zielbotschaften der Schule die Lehrkräfte wahrnehmen (Skaalvik \& Skaalvik, 2017). 
Die inhaltliche Konzeptualisierung der Schul- oder Klassenzielstruktur ist für Schüler(innen) und Lehrkräfte ähnlich. Entsprechende Arbeiten kontrastieren meist zwischen einer Lern- und einer Performanzzielstruktur (Lau \& Nie, 2008; Murayama \& Elliot, 2009; Wolters \& Daugherty, 2007). Eine Vermeidungsperformanzzielstruktur konnte nicht als unabhängig von einer Annäherungsperformanzzielstruktur nachgewiesen werden (Wolters, 2004). Eine starke Schul-Lernzielstruktur betont das Verständnis von Lerngegenständen und persönlicher Entwicklung, fokussiert Verbesserungsmöglichkeiten, betrachtet Fehler als Lernchancen und ist durch eine individuelle oder kriteriale Bezugsnorm geprägt (Ames, 1992; Anderman \& Wolters, 2006; Urdan \& Turner, 2005). Demgegenüber ist eine Schul-Performanzzielstruktur charakterisiert durch eine starke Gewichtung von Leistungsergebnissen anstelle von Einsatz und Verbesserung, sozialen Bezugsnormen und öffentlichen Leistungsrückmeldungen (Skaalvik \& Skaalvik, 2011).

Zu den durch Lehrkräfte wahrgenommenen Schulzielstrukturen gibt es bislang erst wenige empirische Befunde. Erste Arbeiten bestätigten die zugrundeliegende Annahme, dass sich die wahrgenommenen Zielstrukturen auf die korrespondierenden selbstbezogenen Zielpräferenzen der Lehrkräfte auswirkten (Cho \& Shim, 2013), wobei dieser Zusammenhang durch die Selbstwirksamkeitserwartungen der Lehrkräfte moderiert wurde: Lehrkräfte mit hohen Selbstwirksamkeitserwartungen ließen sich in ihrer selbstgezogenen Zielverfolgung weniger von der Schulzielstruktur beeinflussen, wohingegen Lehrkräfte mit geringen Selbstwirksamkeitserwartungen diese stärker übernahmen. Darüber hinaus fanden sich günstige Zusammenhänge einer SchulLernzielstruktur mit beruflicher Zufriedenheit, Zugehörigkeitsgefühlen, Engagement und Selbstwirksamkeitserwartungen und ungünstige Zusammenhänge einer Schul-Performanzzielstruktur mit Zeitdruck, geringerer Zugehörigkeit und emotionaler Erschöpfung der Lehrkräfte (Avanzi et al., 2013; Skaalvik \& Skaalvik, 2011, 2013, 2017).

Zusammenfassend handelt sich bei der Schulzielstruktur um eine wichtige Forschungsrichtung, um kontextuellen Antezedenzien der Lehrkraftmotivationen nachzugehen. Eine spannende Perspektive zukünftiger Forschungsarbeiten besteht darin, sowohl Schüler(innen)- als auch Lehrkraftwahrnehmungen der Zielstruktur zu berücksichtigen. Außerdem erscheint es zur Aufklärung der Schulzielstrukturen gewinnbringend, Unterschieden zwischen verschiedenen Schulen, Schularten und übergeordneten kontextuellen Vorgaben (z. B. Bildungssysteme, Lehrpläne) nachzugehen sowie konkrete kontextuelle Merkmale auf Schulebene selbst, wie etwa die Feedbackkultur oder den Zusammenhalt im Kollegium (vgl. Dickhäuser et al., 2012), zu berücksichtigen. 


\section{Intrinsische und extrinsische Motivation, Bedürfnisbefriedigung im Rahmen der Selbstbestimmungstheorie}

Ein weiterer theoretischer Zugang zur Beschreibung, wie Personen den Wert einer Tätigkeit und deren Ergebnisse (subjektiv) erachten, liegt in der Unterscheidung zwischen intrinsischer Motivation und verschiedenen Formen extrinsischer Motivation, die von Deci und Ryan $(1985,1993)$ in ihrer Selbstbestimmungstheorie der Motivation vorgeschlagen wurde.

Intrinsische Motivation drückt darin die selbstbestimmte und autonome Ausführung von Handlungen, unabhängig von Verstärkungen oder Sanktionen aus und ist durch Interesse, Entdeckungsfreude, Spaß und handlungsinherente Befriedigungen gekennzeichnet (Ryan, 1995).

Extrinsische Motivationen sind im Gegensatz dazu von meist nicht spontan auftretenden Handlungen mit instrumentellen Absichten gekennzeichnet, die dazu dienen, von der Handlung getrennte Konsequenzen zu erlangen (Deci \& Ryan, 1993). Verhalten wird entsprechend dann als extrinsisch motiviert bezeichnet, wenn es durch Anreize gesteuert wird, die außerhalb der Tätigkeit selbst liegen (Rheinberg, 2008). Somit sind extrinsische Motivationen vielfältiger, facettenreicher und häufiger vorzufinden als intrinsische. Sie werden von Deci und Ryan (1985) danach klassifiziert, wie selbstbestimmt bzw. kontrolliert diese sind (d. h. wie viel sie aufgrund von Internalisierungs- und Integrationsprozessen mit persönlichen Wert- und Zielsetzungen der handelnden Personen zu tun haben; vgl. Urhahne, 2002). Eine entsprechend differenzierte Betrachtung ist notwendig, um die Auswirkungen des Werts verschiedener Handlungsfolgen angemessen erklären zu können (vgl. Cerasoli, Nicklin \& Ford 2014).

Bei der externalen Regulation sind Handlungen geprägt von externen Verstärkern und Strafen (z. B. Belohnungen oder Geld), auf die die handelnde Person keinen direkten Einfluss hat. Das aus ihnen resultierende Verhalten kann nicht eigenständig kontrolliert werden. Introjizierte Regulation ist gekennzeichnet durch innere Anstöße und inneren Druck. Handlungen werden bei dieser Motivationsform aus Rücksicht gegenüber anderen durchgeführt oder weil sie als wichtig für die Selbstachtung der eigenen Person eingeschätzt werden. Das Verhalten bedarf zwar keiner externen Anreize, jedoch sind innere Appelle (z. B. Verpflichtungsgefühl) oder innere Verstärker und Bestrafungen für Handlungsfolgen (z. B. Emotionen, Gewissen) notwendig. Diese beiden fremdbestimmten Motivationsformen sind mit reduziertem und oberflächlichem 
Verhalten sowie eingeschränktem Wohlbefinden assoziiert und haben langfristig negative Auswirkungen (zsf. Deci \& Ryan, 2009).

Demgegenüber umfasst die identifizierte Regulation Verhaltensweisen, die als persönlich wichtig oder wertvoll anerkannt werden. Die handelnde Person identifiziert sich mit den zugrunde liegenden Werten, die Handlung passt in ihr Selbstbild und die Handlungsziele werden als persönlich bedeutsam (z. B. nützlich) erachtet und wertgeschätzt. Die integrierte Regulation stellt schließlich die günstigste Form der extrinsischen Motivation dar und ist durch eine ausgeprägte Internalisierung identifizierter Werte gekennzeichnet: Die Handlung wird völlig selbstbestimmt ausgeführt und ihre angenommenen Werte und Ziele stimmen widerspruchsfrei mit der Definition des Selbst überein. Diese beiden letzten, verhältnismäßig autonomen Motivationsformen können ähnlich positive Effekte wie die intrinsische Motivation haben (vgl. Ryan \& Deci, 2000).

Diese verschiedenen Motivationsformen schließen sich nicht gegenseitig aus, sondern können gleichzeitig vorliegen, weswegen es sinnvoll ist, sie hinsichtlich ihres Grads an Selbstbestimmung (d. h. dem subjektiv wahrgenommenen internen Anteil an der Regulierung) zu unterscheiden (Ryan \& Deci, 2000).

Eine Reihe von Arbeiten erbrachte, dass diese unterschiedlichen Formen der Motivation auch bei Lehrkräften vorliegen und sie sich wie theoretisch erwartet hinsichtlich ihres Autonomiegrades unterscheiden lassen (Fernet et al., 2008; Roth, Assort, Kanat-Maymom \& Kaplan, 2007). Die Autor(inn)en fanden umso stärkere Zusammenhänge mit Erfolgsgefühlen, je autonomer die Motivationen waren. Für Belastungserleben berichteten Fernet et al. (2008) ein entsprechend umgekehrtes Bild. Darüber hinaus weisen empirische Befunde darauf hin, dass Lehrkräfte, die selbst davon berichten, dass sie ihren Beruf gerne und mit Freude ausüben (also besonders selbstbestimmt motiviert sind), bessere Unterrichtsqualität (mit einem höheren Ausmaß an autonomieunterstützendem Verhalten) erreichen als jene mit stark kontrollierten Motivationen (Fernet, Guay, Senécal \& Austin, 2012; Pelletier, Legault \& Seguin-Levesque, 2002; Roth et al., 2007; Taylor \& Ntoumanis, 2007; Taylor, Ntoumanis \& Standage, 2008). Darüber hinaus existieren erste Erkenntnisse, dass sich dies zum Teil auch auf die Motivation der Schüler(innen) überträgt (Bakker, 2005; Roth et al., 2007; wobei diese Befunde nicht in allen Arbeiten nachgewiesen werden können, z. B. Bieg, Backes \& Mittag, 2011).

Welche Bedingungsfaktoren begünstigen aber nun den Autonomiegrad der Motivation? Eher autonome Formen der Motivation können zwar, per definitionem, nicht künstlich hervorgerufen werden, jedoch wurden förderliche Bedingungen identifiziert, die Zustände der integrierten oder gar intrinsischen Motivation ermöglichen. 
Deci und Ryan (2000) erachten für selbstbestimmte Motivation psychologische Grundbedürfnisse als besonders bedeutungsvoll, da diese vor allem diejenigen Prozesse beeinflussen, mit deren Hilfe der Mensch autonom handelt. Die Selbstbestimmungstheorie postuliert drei angeborene psychologische Bedürfnisse, die für intrinsische und extrinsische Motivationen gleichermaßen relevant sind: Bedürfnis nach Autonomie (Gefühl, selbst-kongruent handeln zu können), Bedürfnis nach Kompetenzerleben (Gefühl, sich auch persönlich weiterentwickeln und Wirksamkeitserfahrungen machen zu können) und Bedürfnis nach sozialer Eingebundenheit (Gefühl, qualitativ gute soziale Interaktionen mit signifikanten anderen Personen zu haben). Nachhaltige selbstbestimmte Motivation entsteht besonders dann, wenn eine Handlung im Sinne einer Befriedigung der Basic Needs erlebt wird, das Individuum sich folglich als eigenständiger Initiator kompetenten Handelns in einer sozialen Umgebung eingebunden fühlt (s. a. Krapp, 2005).

Erste Forschungsarbeiten untersuchten Bedingungsfaktoren für diese Bedürfnisbefriedigung bei Lehrkräften (zsf. Roth, 2014). Diese erbrachten unter anderem, dass Lehrkräfte, die unsicher sind, welche konkreten Erwartungen die Schulleitung hat, auch über ein eingeschränktes Kompetenzerleben und damit verbunden geringere Erfolgsgefühle berichteten (Fernet, Austin, Trepanier \& Dussault, 2013). Taylor et al. (2008) identifizierten wahrgenommene Zeitbeschränkungen, hohen Evaluations- und Konformationsdruck sowie viele Forderungen seitens der Kolleg(inn)en als Aspekte kontextuellen Drucks, die die Bedürfnisbefriedigung der Lehrkräfte und - darüber vermittelt - ihre selbstbestimmte Motivation reduzierten. Als kontextuelle Stütze wurde demgegenüber von Eyal und Roth (2011) eine Schulleitung identifiziert, die klare Ziele und Visionen formuliert und Lehrkräfte mit wertebasierter, intellektueller Stimulation unterstützt, die Schulvisionen kreativ umzusetzen. Ein solcher Führungsstil stand in einem günstigen Zusammenhang mit der Befriedigung der drei Bedürfnisse, der Selbstbestimmung sowie dem Belastungserleben der Lehrkräfte.

Aufbauend auf diesen ersten Arbeiten erscheint es daher sehr vielversprechend, die Forschung vermehrt darauf zu fokussieren, wie Lehrkräfte in ihrer Bedürfnisbefriedigung unterstützt werden. In Hinblick auf zukünftige Forschungsarbeiten erscheint es ferner wichtig, unterschiedliche Inhalts- und Tätigkeitsaspekte der Motivation von Lehrkräften zu berücksichtigen, da man nicht a priori davon ausgehen kann, dass Lehrkräfte hinsichtlich der verschiedenen Aspekte ihres Berufs gleich motiviert sind (vgl. Mayr, 1998). 


\section{Zusammenfassung und Ausblick}

Im Vergleich zur Forschung über die Motivation von Schüler(inne)n steckt die Forschung zur Lehrkraftmotivation noch in den Kinderschuhen. Gleichzeitig handelt es sich um ein sehr wichtiges Forschungsfeld. Wie der vorliegende Beitrag zeigt, sind die Richtung und die Qualität der Lehrkraftmotivation bedeutsam für das persönliche Lernen der Lehrkräfte, ihre Einstellungen, ihr Unterrichtshandeln und ihr Wohlbefinden - Schlüsselaspekte erfolgreichen Unterrichts. Dies gilt in besonderem Maße in der heutigen Gesellschaft, in der unter anderem gutes Unterrichten als eng mit kontinuierlichem, persönlichem Dazulernen verbunden gilt (Radel, Sarrazin, Legrain \& Wild, 2010; Shulman \& Shulman, 2004) sowie Schutzfaktoren gegenüber hoher Lehrkraftbelastung und -ausfall als maßgeblich erachtet werden (s. a. Watt \& Richardson, 2008). Lehrkraftmotivation zu verstehen, zu erklären und zu unterstützen kann daher als ein zentrales Anliegen der empirischen Bildungsforschung und als wichtige Grundlage für gelingende Schulbildung erachtet werden.

Wie die vorliegende Übersicht über das Forschungsfeld zeigte, setzt die Forschung zur Lehrkraftmotivation an bewährten theoretischen Frameworks und Theorien an, die zuvor primär bei Schüler(inne)n und Studierenden untersucht wurden. Dies kann als große Stärke dieser Forschungsrichtung erachtet werden, da die verwendeten Theorien und Modelle somit einen klaren theoretischen Zugang ermöglichen und zu relativ eindeutigen Begrifflichkeiten und Hypothesen und einer Vergleichbarkeit der Forschungsarbeiten beitrugen (Kaplan, 2014). Gleichwohl zeigte die Übersicht der bisherigen Arbeiten, dass die verwendeten Zugänge systematisch weiterentwickelt und auf den jeweiligen Kontext angepasst wurden (vgl. beispielsweise die inhaltliche Ausdifferenzierung der Selbstwirksamkeitserwartungen, die Hinzunahme von weiteren, im Lehrkontext relevanten Zielklassen, etc.). Dies liegt unter anderem an systematischen Unterschieden zwischen den Lern- und Leistungskontexten von Schüler(inne)n und Studierenden und jenen von Lehrkräften. Während für die ersten beiden Gruppen beispielsweise primär das persönliche Lernen und Leisten im Vordergrund steht und jeweils eng miteinander verknüpft ist, stellt für Lehrende, neben ihrem eigenen, auch das Lernen und Leisten ihrer Schüler(innen) einen zentralen Aspekt ihrer Tätigkeit dar, wobei ihr persönliches Lernen und Leisten oft eher voneinander abgegrenzt sind (z. B. Zeit für eine Fortbildung geht zu Lasten der Vorbereitungszeit für den Unterricht). Dies kann zu Unterschieden in den untersuchten Konstrukten führen: Während sich bei Schüler(inne)n beispielsweise Aufgabenziele auf relativ ähnliche Sachverhalte beziehen wie Lernziele (Aufgaben gut zu erledigen, bedeutet hier in der Regel Lernaufgaben gut zu machen und somit viel zu lernen) und folglich eine ähnlichere Rolle für ihr Erleben und 
Verhalten einnehmen könnten, könnte diese Zielklasse bei Lehrenden (bei deren beruflichen Aufgaben das persönliche Lernen oft nur eine geringe unmittelbare Rolle einnimmt) eine andere Bedeutung haben und sich somit in anderer Weise auf ihr berufliches Erleben und Verhalten auswirken (vgl. Daumiller et al., 2018). Trotz etablierter Konstrukte und Theorien ist somit Vorsicht bei der Interpretation der Befunde zu Lehrkräften und einer Übertragung von Forschungsbefunden aus anderen Bereichen angebracht. Lehrkräfte stellen eine eigenständige und besondere Population dar, die dezidiert untersucht werden muss und spezifische Adaptionen von Motivationskonstrukten benötigt.

Die Übersicht über die verschiedenen Forschungsarbeiten zur Lehrkraftmotivation verwies außerdem darauf, dass verschiedene theoretisch-konzeptuelle Zugänge verwendet werden, um die Intensität und Qualität von Motivation zu beschreiben und zu verstehen. Diese unterschiedlichen Motivationstheorien und -zugänge haben unterschiedliche Annahmen über motivationale Prozesse, betrachten diese aus verschiedenen Perspektiven und mit unterschiedlicher Gewichtung. Um das theoretische Konzept der Lehrkraftmotivation und die Bedingungsfaktoren und Auswirkungen motivationaler Prozesse angemessen zu verstehen, ist somit ein Schema notwendig, das auf der Komplementarität der Annahmen und der Foki der verschiedenen Theorien aufbaut (s. a. Kaplan, 2014; Pintrich, 2003; Reeve \& Su, 2014). Nur durch ein entsprechend differenziertes und umfassendes Motivationsverständnis könnten Unterschiede im Erleben, Denken und Handeln der Lehrkräfte adäquat eingeordnet und die passenden Zugänge und Modelle für theoretische, empirische oder praktische Fragestellungen gewählt werden. Dazu wurde in vorliegendem Beitrag ein Rahmenmodell zur Lehrkraftmotivation vorgestellt (vgl. Abbildung 1). Insbesondere fasst dieses die vorgestellten Forschungsbefunde zusammen und hilft somit zu verstehen, auf welche Art und Weise personelle und kontextuelle Faktoren sich - vermittelt über die erwartungsund wertbezogenen Motivationen der Lehrkräfte - auf deren Kognitionen, Handeln und Affekt auswirken und welche Rolle die spezifische Lern- und Leistungssituation in diesem Gefüge einnimmt.

Nachdem das Unterrichtshandeln als Kernaufgabe der Lehrkräfte erachtet wird (z. B. Kunter et al., 2008; Long \& Woolfolk Hoy, 2006), stellen ultimative abhängige Variablen Merkmale auf Seiten der Schüler(innen) dar. Wie die Übersicht über die bisherigen Forschungsarbeiten aufzeigte, sind Auswirkungen motivationaler Unterschiede seitens der Lehrkräfte auf den Kompetenzzuwachs, die Motivation und die Leistungen der Schüler(innen) naheliegend, bislang jedoch nur in verhältnismäßig wenigen Arbeiten adressiert. Zu deren Erfassung erscheint es notwendig, Quellen außerhalb der Lehrkräfte zu berücksichtigen. Schließlich sind Lehrkraftangaben zu ihrem Unterrichtshandeln und dessen Folgen oft nur gering korreliert mit entsprechenden Angaben ihrer Schüler(innen) oder 
außenstehender Beobachter(innen) (Butler, 2014). Darüber hinaus ist die reziproke und dynamische Natur der Lehrer-Schülerbeziehungen zu berücksichtigen.

Andersherum, als man gemeinhin annehmen könnte, berichteten beispielsweise Holzberger, Philipp und Kunter (2013) in einer Studie mit 155 Lehrkräften, dass die von Schüler(inne)n wahrgenommene kognitive Aktivierung und das Classroom Management der Lehrkräfte sich positiv auf die Entwicklung der LehrkräfteSelbstwirksamkeitserwartungen auswirkte. Zusammenfassend sind somit mehr Forschungsarbeiten notwendig, in denen Resultate und Angaben auf Schülerseite berücksichtigt werden (Klassen et al., 2014) und in denen möglichen Wechselwirkungen mit längsschnittlichen Designs nachgegangen wird.

Eine weitere wichtige Forschungsperspektive liegt in der systematischen Berücksichtigung verschiedener Kontexte. Wie dargelegt wurde, sind Kontextmerkmale ein bedeutsamer Einflussfaktor auf die Motivationen der Lehrkräfte, moderieren jedoch auch deren Effekte auf Erleben und Verhalten derselben. Es lassen sich drei verschiedene Bereiche ausmachen, die in der bisherigen Literatur zu Lehrenden aufgegriffen wurden. Dazu gehören verschiedene Unterrichtsfächer (vgl. Han \& Yin, 2016), unterschiedliche Länder (vgl. Watt \& Richardson, 2017) sowie unterschiedliche Bildungseinrichtungen (vgl. bspw. Daumiller, 2018). Ein explizites Aufgreifen und Thematisieren dieser Kontexte und deren spezifischen Anforderungen und Bedingungen an die Lehrkräfte, ihr pädagogisches Handeln sowie unterschiedliche Voraussetzungen und Erwartungen seitens der Schüler(innen) erscheint eine weitere wichtige Perspektive für ein umfassendes Verständnis der Lehrkraftmotivation, ihrer Bedingungen und Auswirkungen sowie essenziell zur praktischen Nutzbarmachung und Anwendung des Konzepts der Lehrkraftmotivation.

Resümierend stellt die Motivation von Lehrkräften demnach ein verhältnismäßig neues und hochgradig spannendes Forschungsfeld dar, das aus unterschiedlichen theoretischen Zugängen und Perspektiven heraus fundiert untersucht und angewendet werden kann und dem sich wichtige weitere Forschungsperspektiven mit bedeutsamen Implikationen für die Schulbildung bieten. 


\section{Literatur}

Abele, A. E. \& Candova, A. (2007). Prädiktoren des Belastungserlebens im Lehrerberuf. Befunde einer 4-jährigen Längsschnittstudie. Zeitschrift für Pädagogische Psychologie, 21, 107-118. doi:10.1024/1010-0652.21.2.107

Ainley, M., Hidi, S. \& Berndorff, D. (2002). Interest, learning, and the psychological processes that mediate their relationship. Journal of Educational Psychology, 94, 545-561. doi:10.1037/0022-0663.94.3.545

Alexander, P. A. (2008). Charting the course for the teaching profession. The energizing and sustaining role of motivational forces. Learning and Instruction, 18, 483-491. doi:10.1016/j.learninstruc.2008.06.006

Ames, C. (1992). Achievement goals and the classroom motivational climate. In J. L. Meece \& D. H. Schunk (Eds.), Student perceptions in the classroom (pp. 327348). Hillsdale, NJ: Erlbaum.

Anderman, E. M. \& Wolters, C. A. (2006). Goals, values and affect. Influences on student motivation. In P. A. Alexander \& P. H. Winne (Eds.), Handbook of educational psychology (pp. 369-389). Mahwah, N.J: Erlbaum.

Ashton, P. T. \& Webb, R. B. (1986). Making a difference. Teachers' sense of efficacy and student achievement. White Plains, NY: Lonman.

Avanzi, L., Miglioretti, M., Velasco, V., Balducci, C., Vecchio, L., Fraccarolia, F. \& Skaalvik, E. M. (2013). Cross-validation of the Norwegian Teacher's SelfEfficacy Scale (NTSES). Teaching and Teacher Education, 31, 69-78. doi:10.1016/j.tate.2013.01.002

Bakker, A. B. (2005). Flow among music teachers and their students: The crossover of peak experiences. Journal of Vocational Behavior, 66, 26-44. doi:10.1016/j.jvb.2003.11.001

Bandura, A. (1977). Self-efficacy: Toward a unifying theory of behavioral change. Psychological Review, 84, 191-215. doi:10.1037//0033-295x.84.2.191

Bandura, A. (1993). Perceived self-efficacy in cognitive development and functioning. Educational Psychologist, 28, 117-148. doi:10.1207/s15326985ep2802_3

Bandura, A. (1997). Self-efficacy: The exercise of control. New York, NY: W. H. Freeman.

Bandura, A. (2001). Social cognitive theory: An agentic perspective. Annual Review of Psychology, 52, 1-26. doi:10.1146/annurev.psych.52.1.

Barron, K. E. \& Harackiewicz, J. M. (2001). Achievement goals and optimal motivation: Testing multiple goal models. Journal of Personality and Social Psychology, 80, 706-722. doi:10.1037/0022-3514.80.5.706

Bernacki, M., Nokes-Malach, T., Richey, J. E. \& Belenky, D. M. (2016). Science diaries: A brief writing intervention to improve motivation to learn science. Educational Psychology, 36, 26-46.

Baumert, J. \& Kunter, M. (2006). Stichwort: Professionelle Kompetenz von Lehrkräften. Zeitschrift für Erziehungswissenschaft, 9, 469-520. doi:10.1007/s11618-0060165-2 
Baumert, J. \& Kunter, M. (2011). Das Kompetenzmodell von COACTIV. In M. Kunter et al. (Hrsg.), Professionelle Kompetenz von Lehrkräften - Ergebnisse des Forschungsprogramms COACTIV (S. 29-53). Münster: Waxmann.

Benning, K., Daumiller, M., Praetorius, A.-K., Lenske, G., Dickhäuser, O. \& Dresel, M. (2018). Evaluation eines Interventionsansatzes zur Verbesserung von Motivation und motivationsförderlichem Unterrichtshandeln von Lehrkräften auf Basis der Zielorientierungstheorie. Unterrichtswissenschaft. Advanced online publication. doi:10/cswg

Betoret, F. D. (2006). Stressors, self-efficacy, coping resources, and burnout among secondary school teachers in Spain. Educational Psychology, 26, 519-539. doi:10.1080/01443410500342492

Bieg, S., Backes, S. \& Mittag, W. (2011). The role of intrinsic motivation for teaching, teachers' care and autonomy support in students' self - determined motivation. Journal for Educational Research Online, 3(1), 122-140.

Brown, C. G. (2012). A systematic review of the relationship between self-efficacy and burnout in teachers. Educational and Child Psychology, 29(4), 47-63.

Butler, R. (2007). Teachers' achievement goal orientation and association with teachers' help-seeking: Examination of a novel approach to teacher motivation. Journal of Educational Psychology, 99, 241-252. doi:10.1037/0022-0663.99.2.241

Butler, R. (2012). Striving to connect: Extending an achievement goal approach to teacher motivation to include relational goals for teaching. Journal of Educational Psychology, 104, 726-742. doi:10.1037/a0028613

Butler, R. (2014). What teachers want to achieve and why it matters. In P. W. Richardson, S. A. Karabenick \& H. M. G. Watt (Eds.), Teacher motivation: Theory and practice (pp. 20-35). New York, NY: Routledge.

Butler, R. \& Shibaz, L. (2008). Achievement goals for teaching as predictors of students' perceptions of instructional practices and students' help seeking and cheating. Learning and Instruction, 18, 453-467. doi:10.1016/j.learninstruc.2008.06.004

Caprara, G. V., Barbaranelli, C., Steca, P. \& Malone, P. S. (2006). Teachers' self-efficacy beliefs as determinants of job satisfaction and students' academic achievment. A study at the school level. Journal of School Psychology, 44. 473-490. doi:10.1016/j.jsp.2006.09.001.

Cerasoli, C. P., Nicklin, J. M., Ford, M. T. (2014). Intrinsic motivation and extrinsic incentives jointly predict performance: A 40-year meta-analysis. Psychological Bulletin, 140(4), 980-1008. doi:10.1037/a0035661.

Cho, Y. \& Shim, S. S. (2013). Predicting teachers' achievement goals for teaching: The role of perceived school goal structure and teachers' sense of efficacy. Teaching and Teacher Education, 32, 12-21. doi:10.1016/j.tate.2012.12.003

Coladarci, T. (1992). Teachers' sense of efficacy and commitment to teaching. Journal of Experimental Education, 60, 323-337. doi:10.1080/00220973.1992.9943869

Daumiller, M. (2018): Motivation von Wissenschaftlern in Lehre und Forschung Struktur, Eigenschaften, Bedingungen und Auswirkungen selbstbezogener Ziele. Wiesbaden: Springer. 
Daumiller, M., Dickhäuser, O. \& Dresel, M. (2018). University instructors' achievement goals for teaching. Journal of Educational Psychology. Advance online publication. doi:10.1037/edu0000271

Daumiller, M., Grassinger, R., Dickhäuser, O. \& Dresel, M. (2016). Structure and relationships of university instructors' achievement goals. Frontiers in Psychology, 7, 1-14. doi:10.3389/fpsyg.2016.00375

Deci, E. L. \& Ryan, R. M. (1985). Intrinsic motivation and self-determination in human behavior. New York, NY: Springer Science \& Business Media

Deci, E. L. \& Ryan, R. (1993). Die Selbstbestimmungstheorie der Motivation und ihre Bedeutung für die Pädagogik. Zeitschrift für Pädagogik, 39(2), 223-238.

Deci, E. L. \& Ryan, R. (2000). The "what" and "why" of goal pursuits: Human needs and the selfdetermination of behavior. Psychological Inquiry, 11, 269-318. doi:10.1207/s15327965pli1104_02

Deci, E. L. \& Ryan, R. M. (2009). 25 Self-determination theory: A consideration of human motivational universals. In P. J. Corr \& G. Matthews (Eds.), The Cambridge handbook of personality psychology (pp. 441-456). Cambridge, UK: Cambridge University.

de Jesus, S. N. \& Lens, W. (2005). An integrated model for the study of teacher motivation. Applied Psychology, 54, 119-134. doi:10.1111/apps.2005.54.issue-1

Dember, W. N. (1974). Motivation and the cognitive revolution. American Psychologist, 29, 161-168. doi:10.1037/h0035907

Dickhäuser, O., Butler, R. \& Tönjes, B. (2007). Das zeigt doch nur, dass ich's nicht kann. Zeitschrift für Entwicklungspsychologie und Pädagogische Psychologie, 39, 120 126. doi:10.1026/0049-8637.39.3.120

Dickhäuser, O., Nitsche, S., Fasching, M. S. \& Dresel, M. (2012). Kommt es auf die Schule an? Zu Bedeutung von wahrgenommenen Kontextmerkmalen für die beruflichen Zielorientierungen von Lehrkräften. Vortrag auf dem 48. Kongress der Deutschen Gesellschaft für Psychologie (DGPs), Bielefeld.

Dresel, M., Fasching, M. S., Steuer, G., Dickhäuser, O. \& Daumiller, M. (2018). From teachers' personal achievement goals to students' perceptions of classroom goal structures: Via student-directed goals and specific instructional practices. Manuscript submitted for publication.

Dresel, M., Fasching, M. S., Steuer, G., Nitsche, S. \& Dickhäuser, O. (2013). Relations between teachers' goal orientations, their instructional practices and student motivation. Psychology, 7, 572-584. doi:10.4236/psych.2013.47083

Dresel, M. \& Lämmle, L. (2017). Motivation. In T. Götz (Hrsg.), Emotion, Motivation und selbstreguliertes Lernen (2. Aufl.) (S. 80-142). Paderborn: Schöningh.

Dweck, C. S. (1986). Motivational processes affecting learning. American Psychologist, 41, 1040-1048. doi:10.1037//0003-066x.41.10.1040

Eccles, J. S. (1983). Expectancies, values, and academic behaviors. In J. T. Spence (Ed.), Achievement and achievement motivation (pp. 75-146). San Francisco, CA: Freeman. 
Eccles, J. S. (2005). Subjective task value and the Eccles et al. model of achievementrelated choices. In A. J. Elliot, C. S. Dweck \& D. S. Yeager (Eds.), Handbook of competence and motivation (pp. 105-121). New York, NY: Guilford.

Eccles, J. S. (2009). Who am I and what am I going to do with my life? Personal and collective identities as motivators of action. Educational Psychologist, 44, 78-89. doi:10.1080/00461520902832368

Eccles, J. S. \& Wigfield, A. (2002). Motivational beliefs, values, and goals. Annual Review of Psychology, 53, 109-132. doi:10.1146/annurev.psych.53.100901.135153

Edgyed, C. J. \& Short, R. J. (2006). Teacher self-efficacy, burnout, experience and decision to refer a disruptive student. School Psychology International, 27, 462474. doi: $10.1177 / 0143034306070432$

Elliot, A. J. \& Harackiewicz, J. M. (1996). Approach and avoidance achievement goals and intrinsic motivation: A mediational analysis. Journal of Personality and Social Psychology, 70, 461-475. doi:10.1037//0022-3514.70.3.461

Elliot, A. J. \& Hulleman, C. S. (2017). Achievement goals. In A. J. Elliot, C. S. Dweck \& D. S. Yeager (Eds.), Handbook of competence and motivation (pp. 43-60). New York, NY: Guilford .

Elliot, A. J. \& McGregor, H. A. (2001). A $2 \times 2$ achievement goal framework. Journal of Personality and Social Psychology, 80, 501-519. doi:10.1037/00223514.80.3.501

Elliott, E. S. \& Dweck, C. S. (1988). Goals: An approach to motivation and achievement. Journal of Personality and Social Psychology, 54, 5-12. doi:10.1037//00223514.54.1.5

Eren, A. (2012). Prospective teachers' interest in teaching, professional plans about teaching and career choice satisfaction. A relevant framework? Australian Journal of Education, 56, 303-318. doi:10.1037/0022-3514.54.1.5

Eyal, O. \& Roth, G. (2011). Principals' leadership and teachers' motivation: Selfdetermination theory analysis. Journal of Educational Administration, 49, 256275. doi: 10.1108/09578231111129055

Fasching, M. S., Dresel, M., Dickhauser, O. \& Nitsche, S. (2010). Goal orientations of teacher trainees: Longitudinal analysis of magnitude, change and relevance. Journal of Educational Research Online, 2, 9-33. Retrieved from http://www.j-er-o.com/index.php/jero/article/viewFile/117/85

Fernet, C., Austin, S., Trépanier, S.-G. \& Dussault, M. (2013). How do job characteristics contribute to burnout? Exploring the distinct mediating roles of perceived autonomy, competence, and relatedness. European Journal of Work and Organizational Psychology, 22, 123-137. doi:10.1080/1359432X.2011.632161

Fernet, C., Guay, F., Senécal, C. \& Austin, S. (2012). Predicting intraindividual changes in teacher burnout: The role of perceived school environment and motivational factors. Teaching and Teacher Education, 28, 514-525. doi:10.1016/j.tate.2011.11.013 
Fernet, C., Senécal, C., Guay, F., Marsh, H. \& Dowson, M. (2008). The Work Tasks Motivation Scale for Teachers (WTMST). Journal of Career Assessment, 16, 256-279. doi:10.1177/1069072707305764.

Firestone, W. A. \& Pennell, J. R. (1993). Teacher commitment, working conditions, and differential incentive policies. Review of Educational Research, 63, 489-525. doi:10.3102/00346543063004489

Franz, U. (2008). Lehrer- und Unterrichtsvariablen im naturwissenschaftlichen Sachunterricht. Eine empirische Studie zum Wissenserwerb und zur Interessenentwicklung in der dritten Jahrgangsstufe. Bad Heilbrunn: Klinkhardt.

Gibson, S. \& Dembo, M. H. (1984). Teacher efficacy: A construct validation. Journal of Educational Psychology, 76, 569-582. doi:10.1037/0022-0663.76.4.569

Hagen, K. M., Gutkin, T. B., Wilson, C. P. \& Oats, R. G. (1998). Using vicarious experience and verbal persuasion to enhance self-efficacy in pre-service teachers. "Priming the pum" for consultation. School Psychology Quarterly, 13(2), 169178. doi: $10.1037 / \mathrm{h} 0088980$.

Han, J. \& Yin, H. (2016). Teacher motivation: Definition, research development and implications for teachers. Cogent Education, 3, 1-18. doi:10.1080/2331186X.2016.1217819

Han, J., Yin, H. \& Wang, W. (2015). Exploring the relationship between goal orientations for teaching of tertiary teachers and their teaching approaches in china. Asia Pacific Education Review, 16, 537-547. doi:10.1007/s12564-015-9392-7

Han, J., Yin, H. \& Wang, W. (2016). The effect of tertiary teachers' goal orientations for teaching on their commitment: The mediating role of teacher engagement. Educational Psychology, 36, 526-547. doi:10.1080/01443410.2015.1044943

Heckhausen, H. (1987). Jenseits des Rubikon: Der Wille in den Humanwissenschaften. Berlin: Springer.

Heckhausen, H. \& Rheinberg, F. (1980). Lernmotivation im Unterricht, erneut betrachtet. Unterrichtswissenschaft, 8, 7-47.

Heckhausen, H. \& Gollwitzer, P. (1987). Thought contents and cognitive functioning in motivational versus volitional states of mind. Motivation and Emotion, 11, 101120. doi:10.1007/bf00992338

Herranen, J. K., Vesterinen, V. -M. \& Aksela, M. K. (2015). How to measure elementary teachers' interest in teaching chemistry? Chemistry Education Research and Practice, 16, 408-416. doi:10.1039/C4RP00246F

Hidi, S. \& Renninger, K. A. (2006). The four-phase model of interest development. Educational Psychologist, 41, 111-127. doi:10.1207/s15326985ep4102_4

Holzberger, D., Philipp, A. \& Kunter, M. (2013). How teachers' self efficacy is related to instructional quality. A longitudinal analysis. Journal of Educational Psychology, 105(3), 774-786. doi:10.1037/a0032198.

Holland, J. L. (1996). Exploring careers with a typology: What we have learned and some new directions. American Psychologist, 51, 397-406. doi:10.1037/0003-

066X.51.4.397 
Holland, J. L. (1997). Making vocational choices: A theory of vocational personalities and work environments (3rd ed.). Odessa, FL: Psychological Assessment Resources.

Hoyert, M. S. \& O'Dell, C. D. (2006). A brief intervention to aid struggling students: A case of too much motivation? Journal of Scholarship of Teaching and Learning, 6, 1-13. Retrieved from http://josotl.indiana.edu/article/view/1622/1621

Hulleman, C. S., Schrager, S. M., Bodmann, S. M. \& Harackiewicz, J. M. (2010). A metaanalytic review of achievement goal measures: Different labels for the same constructs or different constructs with similar labels? Psychological Bulletin, 136, 422-449. doi:10.1037/a0018947

Janke, S., Nitsche, S. \& Dickhäuser, O. (2015). The role of perceived need satisfaction at work for teachers' work-related learning goal orientation. Teaching and Teacher Education, 47, 184-194. doi:10.1016/j.tate.2015.01.009

Kaplan, A. (2014). Section commentary: Theory and research on teachers' motivation: Mapping an emerging conceptual terrain. In P. W. Richardson, S. A. Karabenick \& H. M. G. Watt (Eds.), Teacher motivation: Theory and practice (pp. 74-88). New York, NY: Routledge.

Kaplan, A. \& Maehr, M. L. (1999). Achievement goals and student well-being. Contemporary Educational Psychology 24, 330-358. doi:10.1006/ceps.1999.0993.

Kelm, J. L., \& McIntosh, K. (2012). Effects of school-wide positive behavior support on teacher self-efficacy. Psychology in the Schools, 49, 137-147. doi:10.1002/pits.20624

Klassen, R. M. \& Chiu, M. M. (2010). Effects on teachers' self-efficacy and job satisfaction: Teacher gender, years of experience, and job stress. Journal of Educational Psychology, 102, 741-756. doi:10.1037/a0019237.

Klassen, R. M. \& Chiu, M. M. (2011). The occupational commitment and intention to quit of practicing and pre-service teachers: Influence of self-efficacy, job stress, and teaching context. Contemporary Educational Psychology, 36, 114-129. doi:10.1016/j.cedpsych.2011.01.002

Klassen, R. M. \& Durksen, T. L. (2014). Weekly self-efficacy and work stress during the teaching practicum: A mixed methods study. Learning and Instruction, 33, 158169. doi:10.1016/j.learninstruc.2014.05.003

Klassen, R. M, Durksen, T. L. \& Tze, V. M. C. (2014). Teachers' self-efficacy beliefs: Ready to move from theory to practice? In P. W. Richardson, S. A. Karabenick \& H. M. G. Watt (Eds.), Teacher motivation: Theory and practice (pp. 100-115). New York, NY: Routledge.

Klassen, R. M. \& Tze, V. M. C. (2014). Teachers' self-efficacy, personality, and teaching effectiveness: A meta-analysis. Educational Research Review, 12, 59-76. doi:10.1016/j.edurev.2014.06.001

Klassen, R. M., Tze, V. M. C., Betts, S. M. \& Gordon K. A. (2011). Teacher efficacy research 1998-2009: Signs of progress or unfulfilled promise? Educational Psychology Review, 23, 21-43. doi:10.1007/s10648-010-9141-8

Kleinbeck, U. (2010). Arbeitspsychologie. Göttingen: Hogrefe. 
Krapp, A. (2001). Pädagogische Psychologie: Ein Lehrbuch. Weinheim: Beltz.

Krapp, A. (2002). Structural and dynamic aspects of interest and development. Theoretical considerations from an ontogenetic perspective. Learning and Instruction, 12, 383-409. doi:10.1016/S0959-4752(01)00011-1

Krapp, A. (2005). Psychologische Bedürfnisse und Interesse. Theoretische Überlegungen und praktische Schlussfolgerungen. In R. Vollmeyer \& J. Brunstein (Hrsg.), Motivationspsychologie und ihre Anwendung (S. 23-38). Stuttgart: Kohlhammer.

Krapp, A. (2009). Pädagogische Psychologie: Lernmotivation und Interesse. In G. Krampen (Hrsg.), Psychologie - Experten als Zeitzeugen (S. 166-179). Göttingen: Hogrefe.

Krapp, A. \& Prenzel, M. (2011). Research on interest in science: theories, methods, and findings. Journal International Journal of Science Education, 33, 27-50. doi:10.1080/09500693.2010.518645

Kunst, E. M., van Woerkom, M. \& Poell, R. F. (2018). Teachers' goal orientation profiles and participation in professional development activities. Vocations and Learning, 11, 91-111. doi:10.1007/s12186-017-9182-y

Kunter, M. (2014): Forschung zur Lehrermotivation. In E. Terhart, H. Bennewitz \& M. Rothland (Hrsg.), Handbuch der Forschung zum Lehrerberuf (698-711). Münster: Waxmann.

Kunter, M., Tsai, Y.-M., Klusmann, U., Brunner, M., Krauss, S. \& Baumert, J. (2008). Students' and mathematics teachers' perceptions of teacher enthusiasm and instruction. Learning and Instruction, 18, 468-482. doi:10.1016/j.learninstruc.2008.06.008

Lange, K. (2010). Zusammenhänge zwischen naturwissenschaftsbezogenem fachspezifisch-pädagogischem Wissen von Grundschullehrkräften und Fortschritten im Verständnis naturwissenschaftlicher Konzepte bei Grundschülerinnen und-schülern. Dissertation. Universität Münster.

Lau, S. \& Nie, Y. (2008). Interplay between personal goals and classroom goal structures in predicting student outcomes: A multilevel analysis of person-context interactions. Journal of Educational Psychology, 100, 15-29. doi:10.1037/00220663.100.1.15

Lin, X., Schwartz, D. L. \& Hatano, G. (2005). Toward teachers' adaptive metacognition. Educational Psychologist, 40, 245-255. doi:10.1207/s15326985ep4004_6

Long, J. F. \& Woolfolk Hoy, A. (2006). Interested instructors. A composite portrait of individual differences and effectiveness. Teaching and Teacher Education, 22, 303-314. doi:10.1016/j.tate.2005.11.001

Lortie, D. (1975). Schoolteacher: A sociological analysis. Chicago, Il: University of Chicago.

Maehr, M. L. \& Zusho, A. (2009). Achievement goal theory: The past, present, and future. In K. R.Wentzel \& A.Wigfield (Eds.), Handbook of motivation at school (pp. 77104). New York, NY: Routledge. 
Mascret, N., Elliot, A. J. \& Cury, F. (2015). The 3 x 2 achievement goal questionnaire for teachers. Educational Psychology, 37, 346-361. doi:10.1080/01443410.2015.1096324

Maslach, C., Jackson, S. E. \& Leiter, M. P. (1996). Maslach burnout inventory manual. Mountain View, CA: CPP.

Mayer, J. D., Faber, M. A. \& Xu, X. (2007). Seventy-five years of motivation measures (1930-2005): A descriptive analysis. Motivation and Emotion, 31, 83-103. doi:10.1007/s11031-007-9060-2

Mayr, J. (1998). Die „Lehrer-Interessen-Skalen“ (LIS). Ein Instrument für Forschung und Laufbahnberatung. In J. Abel (Hrsg.), Pädagogisch-psychologische Interessenforschung in Studium und Beruf(S. 111-125). Münster: Waxmann.

Meece, J. L., Anderman, E. M. \& Anderman, L. H. (2006). Classroom goal structure, student motivation, and academic achievement. Annual Review of Psychology, 57, 487-503. doi:10.1146/annurev.psych.56.091103.070258

Middleton, M. J. \& Midgley, C. (1997). Avoiding the demonstration of lack of ability: An underexplored aspect of goal theory. Journal of Educational Psychology, 89, 710739. doi:10.1037//0022-0663.89. 4.710

Midgley, C., Feldlaufer, H. \& Eccles, J. (1989). Change in teacher efficacy and student self- and task-related beliefs in mathematics during the transition to junior high school. Journal of Educational Psychology, 81, 247-258. doi:10.1037//00220663.81.2.247.

Mitchell, T. R. (1997). Matching motivational strategies with organizational contexts. Research in Organizational Behavior, 19, 57-149.

Möller, Kornelia (2004). Naturwissenschftliches Lernen in der Grundschule. Welche Kompetenzen brauchen Grundschullehrkräfte? In H. Merkens (Hrsg.), Lehrerbildung: IGLU und die Folgen (S. 85-84). Wiesbaden: Verlag für Sozialwissenschaften.

Murayama, K. \& Elliot, A. J. (2009). The joint influence of personal achievement goals and classroom goal structures on achievement-relevant outcomes. Journal of Educational Psychology, 101, 432-447. doi:10.1037/a0014221

Murphy, P. \& Alexander, P. A. (2000). Amotivated exploration of motivation terminology. Contemporary Educational Psychology, 25, 3-53. doi:10.1006/ceps.1999.1019

Myers, N. D. \& Feltz, D. L. (2007). From self-efficacy to collective efficacy in sport. In G. Tenenbaum \& R. C. Eklund (Eds.), Handbook of sport psychology (3rd ed., pp. 799-819). Hoboken, NJ: Wiley.

Nicholls, J. G. (1984). Achievement motivation: Conceptions of ability, subjective experience, task choice, and performance. Psychological Review, 91, 328-346. doi:10.1037//0033-295x.91.3.328

Nitsche, S., Dickhäuser, O., Fasching, M. S. \& Dresel, M. (2011). Rethinking teachers' goal orientations: Conceptual and methodological enhancements. Learning and Instruction, 21, 574-586. doi:10.1016/j.learninstruc.2010.12.001 
Nitsche, S., Dickhäuser, O., Fasching, M. S. \& Dresel, M. (2013). Teachers' professional goal orientations: Importance for further training and sick leave. Learning and Individual Differences, 23, 272-278. doi:10.1016/j.lindif.2012.07.017

Nitsche, S., Praetorius, A.-K., Janke, S., Drexler, K., Fasching, M. S., Dresel, M. \& Dickhäuser, O. (2017). Berufliche Zielorientierungen von Lehrkräften: Entwicklungsbedingungen, Auswirkungen auf berufliche Kompetenzentwicklung, Effekte auf instruktionales Verhalten sowie Lernprozesse und Lernergebnisse von Schülerinnen und Schülern. In C. Gräsel \& K. Trempler (Hrsg.), Entwicklung von Professionalität pädagogischen Personals: Interdisziplinäre Betrachtungen, Befunde und Perspektiven (S. 17-35). Wiesbaden: Springer.

Noordzij, G., van Hooft, E. A. J., van Mierlo, H., van Dam, A. \& Born, M. P. (2013). The effects of a learning-goal orientation training on self-regulation: A field experiment among unemployed job seekers. Personnel Psychology, 66, 723-755. doi:10.1111/peps.12011

O'Connor, R. \& Korr, W. S. (1996). A model for school social work facilitation of teacher self-efficacy and empowerment. Social Work in Education, 18, 45-51. doi:10.1093/cs/18.1.45

Osterwalder, F. (2003). Schatten über der Schule - Schatten über den Lehrenden. Lehrerschelte und ihre historischen Funktionen. Pädagogik, 55(3), 30-33.

Papaioannou, A. \& Christodoulidis, T. (2007). A measure of teachers' achievement goals. Educational Psychology, 27, 349-361. doi:10.1080/01443410601104148

Parker, P. D., Martin, A. J., Colmar, S. \& Liem, G. A. (2012). Teachers' workplace wellbeing: Exploring a process model of goal orientation, coping behavior, engagement, and burnout. Teaching and Teacher Education, 28, 503-513. doi:10.1016/j.tate.2012.01.001

Parajes, F. (1996). Self-efficacy beliefs in academic settings. Review of Educational Research, 66, 543-578. doi:10.3102/00346543066004543

Patrick, B. C.,Hisley, J. \& Kempler, T. (2000). “what's everybody so excited about?”: The effects of teacher enthusiasm on student intrinsic motivation and vitality. The Journal of Experimental Education, 68, 217-236. doi:10.1080/00220970009600093

Pelletier, L. G., Legault, L. \& Séguin-Lévesque, C. (2002). Pressure from above and pressure from below as determinants of teachers' motivation and teaching behaviors. Journal of Educational Psychology, 94, 186-196. doi:10.1037/00220663.94.1.186

Pervin, L. A. (1992). The rational mind and the problem of volition. Psychological Science, 3, 162-164. doi:10.1111/j.1467-9280.1992.tb00018.x

Pintrich, P. R. (1994). Continuities and discontinuities: Future directions for research in educational psychology. Educational Psychologist, 29, 137-148. doi:10.1207/s15326985ep2903_3

Pintrich, P. R. (2000a). An achievement goal theory perspective on issues in motivation terminology, theory, and research. Contemporary Educational Psychology, 25, 92-104. doi:10.1006/ceps.1999.1017 
Pintrich, P. R. (2000b). Multiple goals, multiple pathways: The role of goal orientation in learning and achievement. Journal of Educational Psychology, 92, 544-555. doi:10.1037/0022-0663.92.3.544

Pintrich, P. R. (2003). A motivational science perspective on the role of student motivation in learning and teaching contexts. Journal of Educational Psychology, 95, 667-686. doi:10.1037/0022-0663.95.4.667

Pohlmann, B. \& Möller, J. (2010). Fragebogen zur Erfassung der Motivation für die Wahl des Lehramtsstudiums (FEMOLA). Zeitschrift für Pädagogische Psychologie, 24, 73-84. doi:10.1024/1010-0652/a000005

Posnanski, T. J. (2002). Professional development programs for elementary science teacher. An analysis of teacher self-efficacy beliefs and a professional development model. Journal of Science Teacher Education, 13, 189-220. doi:10.1023/a:1016517100186

Praetorius, A.-K., Nitsche, S., Janke, S., Dickhäuser, O., Drexler, K., Fasching, M. \& Dresel, M. (2014). Here today, gone tomorrow? Revisiting the stability of teachers' achievement goals. Contemporary Educational Psychology. 39, 379387. doi:10.1016/j.cedpsych.2014.10.002

Prenzel, M. \& Schiefele, H. (2001). Motivation und Interesse. In L. Roth (Hrsg.), Pädagogik. Handbuch für Studium und Praxis (2. Aufl., S. 919-930). München: Oldenbourg.

Radel, R., Sarrazin, P., Legrain, P. \& Wild, T. C. (2010). Social contagion of motivation between teacher and student: Analyzing underlying processes. Journal of Educational Psychology, 102, 577-587. doi:10.1037/a0019051

Reeve, J. \& Su, Y. L. (2014). Teacher Motivation. In Gagné, M. (Ed), The Oxford handbook of work engagement, motivation, and self-determination theory (pp. 349-362). New York, NY: Oxford University.

Reichhart, B. (2018). Lehrerprofessionalität im Bereich der politischen Bildung. Wiesbaden: Springer.

Renninger, K. A. \& Hidi, S. (2002). Student interest and achievement: Developmental issues raised by a case study. In A. Wigfield \& J. S. Eccles (Eds.), Development of achievement motivation (pp. 173-195). San Diego, CA: Academic.

Retelsdorf, J., Butler, R., Streblow, L. \& Schiefele, U. (2010). Teachers' goal orientations for teaching: Associations with instructional practices, interest in teaching, and burnout. Learning and Instruction, 20, 34-43. doi:10.1016/j.learninstruc.2009.01.001

Retelsdorf, J. \& Günther, C. (2011). Achievement goals for teaching and teachers' reference norms: Relations with instructional practices. Teaching and Teacher Education, 27, 1111-1119. doi:10.1016/j.tate.2011.05.007

Rheinberg, F. (2008). Motivation. Stuttgart: Kohlhammer.

Richardson, P. W. \& Watt, H. M. G. (2010). Current and future directions in teacher motivation research. In T. C. Urdan \& S. A. Karabenick (Eds.), The decade ahead: Applications and contexts of motivation and achievement (pp. 139-173). Bingley: Emerald. 
Richardson, P. W. \& Watt, H. M. G. (2006). Who chooses teaching and why? Profiling characteristics and motivations across three Australian universities. Asia-Pacific Journal of Teacher Education, 34, 27-56. doi:10.1080/13598660500480290

Richardson, P. W., Karabenick, S. A. \& Watt, H. M. G. (Eds.). (2014). Teacher motivation: Theory and practice. New York, NY: Routledge.

Ross, J. A. (1992). Teacher efficacy and the effects of coaching on student achievment. Canadian Journal of Education, 17, 51-65. doi:10.2307/1495395

Ross, J. A. \& Bruce, C. (2007). Professional development effects on teacher efficacy. Results of randomized field trial. Journal of Educational Research, 101, 50-60. doi:10.3200/JOER.101.1.50-60

Ross, J. A., Hogaboam-Gray, A. \& Hannay, L. (2001). Effects of teacher efficacy on computer skills and computer cognitions of Canadian students in grades K-3. The Elementary School Journal, 102, 141-156. doi:10.1086/499697

Roth, G. (2014). Antecedents and outcomes of teachers' autonomous motivation: A selfdetermination theory analysis. In P. W. Richardson, H. M. G., Watt \& S. A. Karabenick, (Eds.), Teacher motivation: Theory and practice. New York, NY: Routledge.

Roth, G., Assor, A., Kanat-Maymon, Y. \& Kaplan, H. (2007). Autonomous motivation for teaching: How selfdetermined teaching may lead to self-determined learning. Journal of Educational Psychology, 99, 761-744. doi:10.1037/00220663.99.4.761

Runhaar, P., Sanders, K. \& Yang, H. (2010). Stimulating teachers' reflection and feedback asking: An interplay of self-efficacy, learning goal orientation, and transformational leadership. Teaching and Teacher Education, 26, 1154-1161. doi:10.1016/j.tate.2010.02.011

Ryan, R. M. (1995). Psychological needs and the facilitation of integrative processes. Journal of Personality, 63, 397-427. doi:10.1111/j.1467-6494.1995.tb00501.x

Ryan, R. M. \& Deci, E. L. (2000). Intrinsic and extrinsic motivations. Classic definitions and new directions. Contemporary Educational Psychology, 25, 54-67. doi:10.1006/ceps.1999.1020

Rzejak, D. \& Lipowsky, F. (2016): Wer nutzt Lerngelegenheiten engagiert? Die Bedeutung kognitiver und affektiv-motivationaler Merkmale von Lehrpersonen für das Fortbildungsverhalten. Poster präsentiert auf der DGf E-Tagung. Kassel.

Schiefele, H. (1974): Lernmotivation und Motivlernen (2. Aufl.). München: Ehrenwirth.

Schiefele, U., Krapp, A. \& Winteler, A. (1992). Interest as a predictor of academic achievement: A meta-analysis of research. In K. A. Renninger, S. Hidi \& A. Krapp (Eds.), The role of interest in learning and development (pp. 183-212). Hillsdale, NJ: Erlbaum.

Schiefele, U., Streblow, L. \& Retelsdorf, J. (2013). Dimensions of teacher interest and their relations to occupational well-being and instructional practices. Journal for Educational Research Online, 5(1), 7-37.

Schmitz, G. S. \& Schwarzer, R. (2000). Selbstwirksamkeitserwartungen von Lehrern: Längsschnittbefunde mit einem neuen Instrument. Zeitschrift für Pädagogische Psychologie, 14, 12-25. doi:10.1024//1010-0652.14.1.12 
Schmitz, G. S. \& Schwarzer, R. (2002). Individuelle und kollektive Selbstwirksamkeitserwartungen von Lehrern. Zeitschrift für Pädagogik, Beiheft; 44, 192214.

Schunk, D. H. \& Hanson, A. R. (1985). Peer models. Influence on children's self-efficacy and achievment. Journal of Educational Psychology, 77, 313-322. doi:10.1037/0022-0663.77.3.313

Schunk, D., Meece, J. \& Pintrich, P. (2013). Motivation in Education: Theory, research, and applications. Saddle River, NJ: Pearson.

Schunk, D. H. \& Zimmerman, B. J. (2006). Competence and control beliefs. Distinguishing the means and ends. In P. A. Alexander \& P. H. Winne (Eds.), Handbook of educational psychology (pp. 349-367). Mahwah, N.J: Erlbaum.

Schwarzer, R. (1996): Psychologie des Gesundheitsverhaltens (2.Aufl.). Göttingen: Hogrefe

Schwarzer, R. \& Hallum, S. (2008): Perceived teacher self-efficacy as a predictor of job stress and burnout: Mediation analyses. Applied Psychology, 57, 152-171. doi:10.1111/j.1464-0597.2008.00359.x

Schwarzer, R. \& Schmitz, G. S. (1999). Kollektive Selbstwirksamkeitserwartungen von Lehrern. Eine Längsschnittstudie in zehn Bundesländern. Zeitschrift für Sozialpsychologie, 30(4), 262-274.

Schwarzer, R. \& Warner L. M. (2014). Forschung zur Selbstwirksamkeit bei Lehrerinnen und Lehrern. In E. Terhart, H. Bennewitz \& M. Rothland (Hrsg.), Handbuch der Forschung zum Lehrerberuf (S. 662-678). Münster: Waxmann.

Schwerdtfeger, A., Konermann, L. \& Schönhofen, K. (2008). Self-efficacy as a healthprotective resource in teachers? A biopsychological approach. Health Psychology, 27(3), 358-368. doi:10.1037/0278-6133.27.3.358

Senko, C. \& Dawson, B. (2017). Performance-approach goal effects depend on how they are defined: Meta-analytic evidence from multiple educational outcomes. Journal of Educational Psychology, 109, 574-598. doi:10.1037/edu0000160

Shim, S. S., Cho, Y. \& Cassady, J. (2013). Goal structures: The role of teachers' achievement goals and theories of intelligence. The Journal of Experimental Education, 81, 84-104. doi:10.1080/00220973.2011.635168

Shulman, L. S. (1986). Those who understand. Knowledge growth in teaching. Educational Researcher, 15, 4-14. doi:10.3102/0013189X015002004

Shulman, L. J. \& Shulman, J. H. (2004). How and what teachers learn: A shifting perspective. Journal of Curriculum Studies, 36, 257-271. doi:10.1080/0022027032000148298

Skaalvik, E. M. \& Skaalvik, S. (2007). Dimensions of teacher self-efficacy and relations with strain factors, perceived collective teacher efficacy, and teacher burnout. Journal of Educational Psychology, 99, 611-625. doi:10.1037/00220663.99.3.611

Skaalvik, E. M. \& Skaalvik, S. (2011). Teacher job satisfaction and motivation to leave the teaching profession: Relations with school context, feeling of belonging, and emotional exhaustion. Teaching and Teacher Education, 27, 1029-1038. doi:10.1016/j.tate.2011.04.001 
Skaalvik, E. M. \& Skaalvik, S. (2013). Teachers' perceptions of the school goal structure: Relations with teachers' goal orientations, work engagement, and job satisfaction. International Journal of Education Research, 62, 199-209. doi:10.1016/j.ijer.2013.09.004

Skaalvik, E. M. \& Skaalvik, S. (2017). Still motivated to teach? A study of school context variables, stress and job satisfaction among teachers in senior high school. Social Psychology of Education, 20, 15-37. doi:10.1007/s11218-016-9363-9

Spiel, C. \& Schober, B. (2003). Lebenslanges Lernen als Ziel: Zur systematischen Förderung von Bildungsmotivation. Erziehung und Unterricht, 9/10, 1282-1293.

Sternberg, R. J. \& Horvath, J. A. (1995). A prototype view of expert teaching. Educational Researcher, 24, 9-17. doi:10.3102/0013189X024006009

Stipek, D. J., Givvin, K. B., Salmon, J. M. \& MacGyvers, V. L. (2001). Teachers' beliefs and practices related to mathematics instruction. Teaching and Teacher Education, 17, 213-226. doi:10.1016/S0742-051X(00)00052-4

Taylor, I. M. \& Ntoumanis, N. (2007). Teacher motivational strategies and student selfdetermination in physical education. Journal of Educational Psychology, 99, 747760. doi:10.1037/0022-0663.99.4.747

Taylor, I. M., Ntoumanis, N. \& Standage, M. (2008). A self-determination theory approach to understanding the antecedents of teachers' motivational strategies in physical education. Journal of Sport \& Exercise Psychology, 30, 75-94. doi:10.1123/jsep.30.1.75

Terhart, E., Bennewitz, H. \& Rothland, M. (2014). Handbuch der Forschung zum Lehrerberuf (2. Aufl.). Münster: Waxmann.

Terhart, E. (2000). Qualität und Qualitätssicherung im Schulsystem. Hintergründe Konzepte - Probleme, Zeitschrift für Pädagogik, 46(6), 809-829.

Thoonen, E. E. J., Sleegers, P. J. C., Oort, F. J., Peetsma, T. T. D. \& Geijsel, F. P. (2011). How to improve teaching practices: The role of teacher motivation, organizational factors, and leadership practices. Educational Administration Quarterly, 47, 496536. doi:10.1177/0013161X11400185

Tönjes, B. \& Dickhäuser, O. (2009). Längsschnittliche Effekte von Zielorientierungen auf Dimensionen des beruflichen Belastungserlebens im Lehrberuf. Zeitschrift für Entwicklungspsychologie und pädagogische Psychologie, 41, 79-86. doi:10.1026/0049-8637.41.2.79

Tschannen-Moran, M. \& Barr, M. (2004). Fostering student learning. The relationship of collective teacher efficacy and student achievement. Leadership and Policy in Schools, 3, 189-209. doi:10.1080/15700760490503706

Tschannen-Moran, M. \& Woolfolk Hoy, A. (2001). Teacher efficacy: Capturing an elusive construct. Teaching and Teacher Education, 17, 783-805. doi:10.1016/S0742-051X(01)00036-1

Tschannen-Moran, M. \& Woolfolk Hoy, A. (2007). The different antecedents of selfefficacy beliefs of novice and experienced teachers. Teaching and Teacher Education, 23, 944-956. doi:10.1016/j.tate.2006.05.003 
Tschannen-Moran, M., Woolfolk Hoy, A. \& Hoy, W. K. (1998). Teacher efficiacy. Its meaning and measure. Review of Educational Research, 68, 202-248. doi:10.3102/00346543068002202

Urdan, T. \& Midgley, C. (2003). Changes in the perceived classroom goal structure and pattern of adaptive learning during early adolescence. Contemporary Educational Psychology, 28, 524-551. doi:10.1016/S0361-476X(02)00060-7

Urdan, T. \& Turner, J. C. (2005). Competence motivation in the classroom. In A. J. Elliot \& C. S. Dweck (Eds.), Handbook of competence and motivation (pp. 297-317). New York, NY: Guilford.

Urhahne, D. (2002): Motivation und Verstehen. Waxmann: Münster.

Wang, H., Hall, N. C., Goetz, T. \& Frenzel, A. C. (2017). Teachers' goal orientations: Effects on classroom goal structures and emotions. British Journal of Educational Psychology, 87, 90-107. doi:10.1111/bjep.12137

Warner, L. M. \& Schwarzer, R. (2009). Selbstwirksamkeit bei Lehrkräften. In O. ZlatkinTroitschanskaia (Hrsg.), Lehrprofessionalität. Bedingungen, Genese, Wirkungen und ihre Messung (S. 629-640). Weinheim, Basel: Beltz.

Watt, H. M. G. \& Richardson, P. W. (2007). Motivational factors influencing teaching as a career choice: Development and validation of the FIT-choice scale. The Journal of Experimental Education, 75, 167-202. doi:10.3200/JEXE.75.3.167-202

Watt, H. M. G. \& Richardson, P. W. (2008). Motivation for teaching. Learning and Instruction, 18, 405-407. doi:10.1016/j.learninstruc.2008.06.009

Watt, H. M. G. \& Richardson, P. W. (2012). An introduction to teaching motivations in different countries: Comparisons using the FIT-Choice scale. Asia-Pacific Journal of Teacher Education, 40, 185-197. doi:10.1080/1359866X.2012.700049

Watt, H. M. G. \& Richardson, P. W. (2014). Why people choose teaching as a career: An expectancy-value approach to understanding teacher motivation. In P. W. Richardson, S. A. Karabenick \& H. M. G. Watt (Eds.), Teacher motivation: Theory and practice (pp. 100-115). New York, NY: Routledge

Watt, H. M. \& Richardson, P. W. (2017). Teacher motivation. In J. D.Wright (Ed.), International encyclopedia of the social and behavioral sciences (pp. 64-71). San Francisco, CA: Elsevier.

Weiner, B. (1985). An attributional theory of achievement motivation and emotion. Psychological Review, 92, 548-573. doi:10.1037//0033-295x.92.4.548

Wigfield, A. \& Cambria, J. (2010). Students' achievement values, goal orientations, and interest: Definitions, development, and relations to achievement outcomes. Developmental Review, 30, 1-35. doi:10.1016/j.dr.2009.12.001

Wigfield, A., Eccles, J., Schiefele, U., Roester, R. W. \& Davis-Kean, P. (2006). Development of achievment motivation. In N. Eisenberg, W. Damon \& R. M. Lerner (Eds.), Social, emotional, and personality development (pp. 933-1002). Hoboken, N.J: John Wiley \& Sons.

Wolters, C. A. (2004). Advancing achievement goal theory: Using goal structures and goal orientations to predict students' motivation, cognition, and achievement. Journal of Educational Psychology, 96, 236-250. doi:10.1037/00220663.96.2.236 
Wolters, C. A. \& Daugherty, S. G. (2007). Goal structures and teachers' sense of efficacy. Their relation and association to teaching experience and academic level. Journal of Educational Psychology, 99, 181-193. doi:10.1037/0022-0663.99.1.181

Woolfolk Hoy, A. \& Spero, R. B. (2005). Changes in teacher efficacy during the early years of teaching. A comparison of four measures. Teaching and Teacher Education, 21, 343-356. doi:10.1016/j.tate.2005.06.010

Wudy, D.-T. \& Jerusalem, M. (2011). Die Entwicklung von Selbstwirksamkeit und Belastungserleben bei Lehrkräften. Psychologie in Erziehung und Unterricht, 58, 254-267. doi:10.2378/peu2011.art16d

Zimmerman, B. J. \& Cleary, T. J. (2006). Adolescents' development of personal agency. The role of self-efficacy beliefs and self-regulatory skill. In F. Pajares \& T. C. Urdan (Eds.), Self-efficacy beliefs of adolescents (pp. 45-69). Greenwich, CT: IAP.

Zimmerman, B. J. \& Kitsantas, A. (2002). Acquiring writing and self-regulatory skill through observation and emulation. Journal of Educational Psychology, 94(4), 660-668. 